\title{
Bootstrap current and parallel ion velocity in imperfectly optimized stellarators
}

\author{
Peter J. Catto ${ }^{1, \dagger}$ and Per Helander ${ }^{2}$ \\ ${ }^{1}$ Plasma Science and Fusion Center, Massachusetts Institute of Technology, Cambridge, MA 02139, USA \\ ${ }^{2}$ Max-Planck-Institut für Plasmaphysik, 17491 Greifswald, Germany
}

\begin{abstract}
A novel derivation of the parallel ion velocity, and the bootstrap and Pfirsch-Schlüter currents in an imperfectly optimized (that is, almost omnigenous) stellarator magnetic field, $\overrightarrow{\mathrm{B}}$, is presented that somewhat more generally recovers expressions completely consistent with previous analytic results. However, it is also shown that, when the conventional radially local form of the drift kinetic equation is employed, the flow velocity and the bootstrap current acquire a spurious contribution proportional to $\omega / v$, where $\omega$ denotes the $\overrightarrow{\mathrm{E}} \times \overrightarrow{\mathrm{B}}$ rotation frequency (due to the radial electric field $\overrightarrow{\mathrm{E}}$ ) and $v$ the collision frequency. This contribution is particularly large in the $\sqrt{v}$ regime and at smaller collisionalities, where $\omega / v \gtrsim 1$, and is presumably present in most numerical calculations, but it disappears if a more accurate drift kinetic equation is used.
\end{abstract}

\section{Introduction}

In stellarators and tokamaks, the flow velocity of each plasma species along the magnetic field is affected by the cross field (radial) derivatives of the density, electrostatic potential and temperature, and so is, therefore, the parallel current. The existence of this "bootstrap" current is of great practical importance, but in stellarators it is still incompletely understood. It can be calculated numerically from the drift kinetic equation, but analytical expressions have only been derived in the so called $1 / v$ regime of moderately small collisionality (Shaing et al. 1989; Helander et al. 2011 \& 2017), the omnigenous limit (Landreman \& Catto 2012), and, more recently, for a Lorentz collision operator extension into the $\sqrt{v}$ regime of lower collisionalty (Helander et al. 2017). In all these regimes, the theory predicts a current (or parallel flow velocity) that is independent of collisionality, but numerical simulations (Beidler et al. 2011; Kernbichler et al. 2016) suggest otherwise down to very small collisionalities. They also find that the bootstrap current depends sensitively on the radial electric field - an effect not contained in the analytical expressions.

In the present paper, a possible reason for radial electric field sensitivity is proposed. It is shown that the standard drift kinetic equation solved by most numerical codes leads to a spurious term in the parallel flow velocity proportional to $\omega / \nu$, where $\omega$ denotes the poloidal $\overrightarrow{\mathrm{E}} \times \overrightarrow{\mathrm{B}}$ rotation frequency and $v$ the collision frequency. (Here $\vec{E}$ denotes the radial electric field and $\vec{B}$ the magnetic field strength.) This contribution to the bootstrap current is particularly important in the $\sqrt{v}$ regime and at smaller collisionalities, where $\omega / v>>1$, and does not appear in analytical formulas, but it will be present in most numerical simulations. However, it is also shown that this term proportional to $\omega / v$ is incorrect. It results from the use of a radially local approximation of 
the drift kinetic equation, which is nearly always employed in the neoclassical theory of stellarators, both in analytical theory and in numerical simulations. It usually seems to be admissible, but apparently fails for parallel flows.

In the absence of turbulence, optimized stellarators attempt to confine all collisionless charged particle orbits by clever design of the magnetic equilibrium. If the magnetic field strength B on a flux surface (labeled by the poloidal flux function $\psi$ ) depends on the toroidal $(\zeta)$ and poloidal $(\vartheta)$ angles in a single linear combination $(\mathrm{M} \vartheta-\mathrm{N} \zeta)$,

$$
\mathrm{B}=\mathrm{B}(\psi, \mathrm{M} \vartheta-\mathrm{N} \xi),
$$

the field is said to be quasisymmetric (Nührenberg \& Zille 1988, Boozer 1995), as in the Helically Symmetric Experiment (HSX) stellarator (Anderson et al. 1995), and the collisionless orbits are all confined. Here $\mathrm{M}$ and $\mathrm{N}$ are integers corresponding to the number of times a line of constant B on a flux surface must traverse the torus toroidally and poloidally, respectively, before closing on itself. Quasisymmetric behavior is thought to be possible on only an isolated flux or constant pressure surface (Garren \& Boozer 1991; Plunk \& Helander 2018; Landreman \& Sengupta 2018; Landreman et al. 2019). More generally, confined collisionless charged particle orbits are possible if the magnetic field has the property normally referred to as omnigeneity (Cary \& Shasharina 1997a\&b), with the quasi-isodynamic behavior of the Wendelstein 7-X (W7-X) stellarator (Nührenberg 2010; Beidler et al. 1990; Grieger et al. 1992) being the most notable example (although it is only approximately realized). (Presumably this more general property can also only be satisfied on or in the vicinity of a single flux surface.) Using $\psi$, $\alpha \equiv \zeta-q \vartheta$, and $\mathrm{B}$ variables, with $\mathrm{q}$ the safety factor, and $\overrightarrow{\mathrm{B}}$ given by the Clebsch representation

$$
\overrightarrow{\mathrm{B}}=\mathrm{B} \overrightarrow{\mathrm{b}}=\nabla \alpha \times \nabla \psi,
$$

a magnetic flux suface is omnigenous when the local condition

$$
\frac{\partial}{\partial \alpha} \Sigma \frac{1}{|\vec{b} \cdot \nabla \mathrm{B}|}=0
$$

is satisfied, where $\Sigma$ denotes a sum over both branches adjacent to the magnetic well. This condition, first found by Cary \& Shasharina (1997a,b), requires that the sum of the incremental lengths $\mathrm{d} \ell=\mathrm{dB} / \overrightarrow{\mathrm{b}} \cdot \nabla \mathrm{B}$ associated with the same $\mathrm{B}$ on each side of the well be alpha independent [for a more detailed explanation see Helander (2014) following Eq. (79)]. Omnigeneity is just the condition required to make the second adiabatic invariant,

$$
\mathrm{J} \equiv(\mathrm{Mc} / \mathrm{Ze}) \oint_{\alpha} \mathrm{d} \ell \mathrm{v}_{\|},
$$

independent of $\alpha$,

$$
\frac{\partial \mathrm{J}}{\partial \alpha}=\frac{\mathrm{Mc}}{\mathrm{Ze}} \oint_{\alpha} \mathrm{dBv}_{\|} \frac{\partial}{\partial \alpha} \Sigma \frac{1}{|\overrightarrow{\mathrm{b}} \cdot \nabla \mathrm{B}|},
$$

where the subscript $\alpha$ on an integral is a reminder that it is to be held fixed while performing the $\mathrm{B}$ integration. Here $\mathrm{Z}$ and $\mathrm{M}$ are the species charge number and mass, while $\mathrm{c}$ is the speed of light and e is the charge on a proton. Stellarator optimization schemes try to approach these idealized limits over a large fraction of the cross section (Henneberg et al. 2019), while 
maintaining good stability properties and avoiding substantial turbulent transport, because significant departures from omnigeneity result in unacceptably large collisional transport.

The neoclassical properties of perfectly omnigenous fields are well understood (Helander \& Nührenberg 2009; Landreman \& Catto 2012), and the effect of departures from omnigeneity on radial particle and heat transport are becoming better documented as simulations and analysis improve. However, understanding of the effect of departures from omnigeneity on the bootstrap current at low collisionalities is less clear. It is likely that transitional particles that spend time trapped in multiple wells are responsible for much of the puzzling behavior as the radial electric field vanishes, and therefore must also be responsible for some of the sensitivity to the radial electric field observed in simulations (Beidler et al. 2011; Kernbichler et al. 2016) but not exhibited by the analytic expressions (Shaing et al. 1989; Helander et al. 2011; Landreman \& Catto 2012; Helander et al. 2017). However, the procedure used here also finds that in the presence of an $\vec{E} \times \vec{B}$ drift tangential to the magnetic surfaces there is a spurious modification to the ion flow that becomes large at low collisionality on weakly non-omnigenous flux surfaces. It introduces a different sensitivity to the radial electric field in both the bootstrap current and the parallel flow velocity and arises when the full magnetic plus electric drift is replaced by only the $\overrightarrow{\mathrm{E}} \times \overrightarrow{\mathrm{B}}$ drift as in most simulations (Beidler et al.2011; Kernbichler et al. 2016).

When the full drift is retained the bootstrap current and parallel ion flow expressions found here are completely consistent with the moderate or $1 / v$ collisionality results of (Shaing et al. 1989; Helander et al. 2011 \& 2017), the omnigeous limit of (Landreman \& Catto 2012), and the moderate $(1 / v)$ and weak $(\sqrt{v})$ collisionality limits of (Helander et al. 2017). As in these earlier treatments the expressions found here for the bootstrap current and parallel ion flow are independent of collision frequency. They are obtained by a very different procedure that is valid in the $\sqrt{v}$ and standard $1 / v$ regimes of collisionality (Galeev et al. 1969; Ho \& Kulsrud 1987; Calvo et al. 2017), and contain slightly more explicit and compact geometrical coefficients than (Shaing et al. 1989; Helander et al. 2011 \& 2017). It is only when the full drift term term is approximated as in simulations that spurious effects arise. The erroneous effects are considered separately in the hope that they will provide some insight into the behavior of the simulations at very small collisionalities.

The analytic expressions for the bootstrap current and parallel flow derived here are also valid in the superbanana plateau regime of collisionality (Shaing 2015; Calvo et al. 2017), where the tangential magnetic drift frequency vanishes at some pitch angle. This regime is considered in only one published neoclassical stellarator simulation (Beidler et al. 1995) as it is not of interest for the background ions unless the $\vec{E} \times \vec{B}$ drift is smaller than the magnetic drift.

The next section discusses ambipolarity and radial transport in weakly non-optimized stellarators. Then section 3 introduces notation, evaluates drifts, and discusses transit and flux surface averages. Important equilibrium properties are discussed in section 4. Section 5 reformulates stellarator drift kinetics to extract the terms odd in the parallel velocity as the lowest order corrections to the Maxwellian. In section 6 the drift kinetic equation is solved properly by retaining the full, nonlocal effects of the magnetic plus electric drifts. These results are employed 
in section 7 to evaluate the parallel ion flow velocity and parallel currenty density. At low collisionality, the specious tangential $\overrightarrow{\mathrm{E}} \times \overrightarrow{\mathrm{B}}$ term retained in most similations and for analytic radial transport evaluations is considered and found to give rise to an incorrect modification of the ion flow velocity and bootstrap current, which is evaluated in section 8 . A brief discussion follows in section 9 .

\section{Ambipolarity and radial transport}

To treat collisional transport in a non-quasisymmetric stellarator it is normally assumed that the electrons and ions are in the $1 / v$ and $\sqrt{v}$ regimes, respectively. In both regimes collisions must be retained, but ion streaming is smaller than electron streaming so the ions are sensitive to the tangential $\vec{E} \times \vec{B}$ drift and the electrons are not. Consequently, as $v$ gets small, the electron diffusivities increase as $1 / v$, while the ion diffusivities decrease as $\sqrt{v}$ (Galeev et al. 1969; Ho \& Kulsrud 1987). The radial ion and electron particle losses balance and set the electric field to maintain ambipolarity. Consequently, both collisionality regimes must be considered when evaluating the bootstrap current.

Ambipolar operation requires considering this mixed collisionality regime where the lowest order radial electric field, $\overrightarrow{\mathrm{E}} \simeq-\nabla \Phi(\psi)$, is roughly at the ion diamagnetic level (to bring ion transport down to the electron level), given by the estimate

$$
\operatorname{Zen}_{\mathrm{i}} \partial \Phi / \partial \psi \sim-\mathrm{T}_{\mathrm{i}} \partial \mathrm{n}_{\mathrm{i}} / \partial \psi
$$

with the poloidal flux function $\psi$ related to the toroidal flux function $\Psi$ by $\mathrm{d} \Psi=\mathrm{qd} \psi$, and the safety factor $q$ and rotational transform $\iota$ related by $\iota q=1$. The tangential $\vec{E} \times \vec{B}$ drift frequency is then

$$
\omega_{\mathrm{E}} \equiv-\overrightarrow{\mathrm{v}}_{\mathrm{E}} \cdot \nabla \alpha=\mathrm{c} \frac{\partial \Phi}{\partial \psi} \sim-\frac{\mathrm{cT}}{\mathrm{Zen}_{\mathrm{i}}} \frac{\partial \mathrm{n}_{\mathrm{i}}}{\partial \psi} \sim \frac{\mathrm{v}_{\mathrm{i}}^{2}}{\Omega_{\mathrm{i}} \mathrm{a}^{2}}=\frac{\rho_{\mathrm{i}}}{\mathrm{a}^{2}} \mathrm{v}_{\mathrm{i}},
$$

while the $\nabla B$ drift, $\vec{v}_{\mathrm{VB}} \sim \rho_{\mathrm{i}} \mathrm{v}_{\mathrm{i}} / \mathrm{R}$, results in a tangental drift frequency of

$$
\omega_{\mathrm{VB}} \equiv-\overrightarrow{\mathrm{v}}_{\mathrm{VB}} \cdot \nabla \alpha \sim \rho_{\mathrm{i}} \mathrm{v}_{\mathrm{i}} / \mathrm{aR},
$$

where $\rho_{\mathrm{i}}$ and $\mathrm{v}_{\mathrm{i}}$ are the ion gyroradius and thermal speed, $\Omega_{\mathrm{i}}=\mathrm{ZeB} / \mathrm{M}_{\mathrm{i}} \mathrm{c}$, and $\mathrm{r}$, a, and $\mathrm{R}$ are the distance from the magnetic axis, the nominal radial scale, and the major radius. The ion charge number and mass are $Z$ and $\mathrm{M}_{\mathrm{i}}$. Normally $\omega_{\mathrm{E}} \sim R \omega_{\nabla \mathrm{B}} / \mathrm{a}>>\omega_{\nabla \mathrm{VB}}$, so the magnetic drift is often neglected as small. Then, there is no superbanana plateau regime as the vanishing of the combined magnetic and electric drift is unlikely for the background ions (at any pitch angle).

As already noted, when all collisionsless orbits are confined, a stellarator is referred to as omnigenous, and the special case of the magnitude of the magnetic field B depending on only a single helical variable is referred to as quasisymmetry. For an omnigenous, but nonquasisymmetric, stellarator, ambipolarity requires that the radial electric field reduce the ion particle transport to the typically smaller electron level by setting it at the ion diamagnetic level as already estimated. More precisely, the ion root is given by (Landreman \& Catto 2012)

$$
\operatorname{Zen}_{\mathrm{i}} \frac{\partial \Phi}{\partial \psi} \simeq-\frac{\partial \mathrm{p}_{\mathrm{i}}}{\partial \psi}+1.17 \mathrm{n}_{\mathrm{i}} \frac{\partial \mathrm{T}_{\mathrm{i}}}{\partial \psi} .
$$


As flux surfaces become non-omnigenous the ion particle diffusivity enters the $\sqrt{v}$ regime and decreases as the collisionality is reduced. The electron particle diffusivity increases as $1 / v$ as the tangential drift is unimportant. At some point the omnigenous ion root may be altered and become sensitive to collisions as $\partial \Phi / \partial \psi$ becomes small and it transitions to the electron root,

$$
\text { en } \mathrm{e}_{\mathrm{e}} \partial \Phi / \partial \psi \sim \mathrm{T}_{\mathrm{e}} \partial \mathrm{n}_{\mathrm{e}} / \partial \psi
$$

to keep the electron particle transport at the now lower ion level. If a change in the sign of $\partial \Phi / \partial \psi$ occurs, then there are flux surfaces with very small $\overrightarrow{\mathrm{E}} \times \overrightarrow{\mathrm{B}}$ drift where the $\nabla \mathrm{B}$ drift matters (Matsuoka et al. 2015). As the $\nabla B$ drift vanishes at some pitch angle, there can be flux surfaces for which superbanana plateau transport becomes important. The treatment here allows for this possibility, but many simulations do not (Beidler et al. 2011; Kernbichler et al. 2016).

Typically the $1 / v, \sqrt{v}$, and superbanana plateau collisionality regimes are evaluated by a transit averaged kinetic equation for the trapped (subscript $\mathrm{t}$ ) particles of the form,

$$
\overline{\overrightarrow{\mathrm{v}}_{\mathrm{d}} \cdot \nabla \psi} \frac{\partial \mathrm{f}_{0}}{\partial \psi}+\overline{\overrightarrow{\mathrm{v}}_{\mathrm{d}} \cdot \nabla \alpha} \frac{\partial \overline{\mathrm{h}}_{\mathrm{t}}}{\partial \alpha}=\overline{\mathrm{C}\left\{\overline{\mathrm{h}}_{\mathrm{t}}\right\}},
$$

with $f_{0}$ a Maxwellian and $\bar{h}_{t}$ a trapped particle modification to it. The overbar notation indicates transit or bounce average and will be defined carefully in the next section. The tangential drift frequency in the $\sqrt{v}$ regime is normally assumed to be just due to the $\overrightarrow{\mathrm{E}} \times \overrightarrow{\mathrm{B}}$ drift,

$$
\overrightarrow{\mathrm{v}}_{\mathrm{d}} \cdot \nabla \alpha \rightarrow \overrightarrow{\mathrm{v}}_{\mathrm{E}} \cdot \nabla \alpha=-\omega(\psi)=-\mathrm{c} \partial \Phi / \partial \psi,
$$

and is often assumed to be a flux function. Only in the superbanana plateau regime is the magnetic drift retained in $\overrightarrow{\mathrm{v}}_{\mathrm{d}} \cdot \nabla \alpha$.

In the transit averaged equation, only the radial drift term $\overline{\overrightarrow{\mathrm{v}}_{\mathrm{d}} \cdot \nabla \psi} \simeq \overrightarrow{\mathrm{v}}_{\nabla \mathrm{B}} \cdot \nabla \psi$ need retain the departure from omnigeneity. Two basic approximations have been made in Eq. (2.7). The radial component of the drift acting on $\bar{h}_{t}$ has been neglected, and the tangential part of the drift has been approximated by its $\overrightarrow{\mathrm{E}} \times \overrightarrow{\mathrm{B}}$ component. Although these approximations are common in neoclassical stellarator theory, it is shown later that they can, in fact, be misleading and lead to errors in the parallel flows.

For the electrons the tangential drift is negligible $\left(\omega<<v_{\mathrm{e}}\right)$, giving

$$
\overline{\overrightarrow{\mathrm{v}}}_{\nabla \mathrm{B}} \cdot \nabla \psi \partial \mathrm{f}_{0 \mathrm{e}} / \partial \psi \sim \overline{\mathrm{C}\left\{\overline{\mathrm{h}}_{\mathrm{te}}\right\}} \sim v_{\mathrm{e}} \overline{\mathrm{h}}_{\mathrm{te}},
$$

$\overline{\mathrm{h}}_{\mathrm{te}} / \mathrm{f}_{0 \mathrm{e}} \propto 1 / v_{\mathrm{e}}$, and electron diffusivitities proportional to $1 / v_{\mathrm{e}}$, with $v_{\mathrm{e}}$ the electron collision frequency. The tangential drift frequency $\omega$ matters for the ions $\left(\omega>>v_{i}\right)$. In the $\sqrt{v}$ regime tangential and radial drift balance for the ions giving $\overline{\mathrm{h}}_{\mathrm{ti}} / \mathrm{f}_{0 \mathrm{i}} \propto 1 / \omega$, except in a the narrow boundary layer of width $\left(v_{i} / \omega\right)^{1 / 2}$ at the trapped-passing boundary. This boundary layer in pitch angle, $\lambda=2 \mu \mathrm{B}_{0} / \mathrm{v}^{2}$, is required to make $\mathrm{f}$ vanish and its width is set by $\omega \partial \overline{\mathrm{h}}_{\mathrm{ti}} / \partial \alpha \sim v_{\mathrm{i}} \partial^{2} \overline{\mathrm{h}}_{\mathrm{ti}} / \partial \lambda^{2}$. As its width is narrower than the usual trapped bounday layer width $\varepsilon^{1 / 2}$, the collisionality is assumed weak enough to satisfy $v_{\mathrm{i}} / \omega<<\varepsilon$ or

$$
\rho_{\mathrm{i}} / \mathrm{a}>\mathrm{R} v_{\mathrm{i}} / \mathrm{v}_{\mathrm{i}}=\mathrm{R} / \lambda
$$

where $\lambda$ is the mean free path, with $\lambda \sim \mathrm{v}_{\mathrm{i}} / v_{\mathrm{i}} \sim \mathrm{v}_{\mathrm{e}} / v_{\mathrm{e}}$ and $\mathrm{v}_{\mathrm{e}}$ the electron thermal speed. 
For $\mathrm{T}_{\mathrm{i}}=10 \mathrm{keV}, \mathrm{n}_{\mathrm{i}}=10^{14} \mathrm{~cm}^{-3}$, and $\mathrm{B}=5 \mathrm{~T} ; \mathrm{v}_{\mathrm{i}} \simeq 10^{8} \mathrm{~cm} / \mathrm{sec}, \mathrm{v}_{\mathrm{i}} \simeq 50 \mathrm{sec}^{-1}$, and $\rho_{\mathrm{i}} \simeq 0.3 \mathrm{~cm}$. Then $\mathrm{a}=100 \mathrm{~cm}$ and $\mathrm{R}=1000 \mathrm{~cm}$ give $\mathrm{R} v_{\mathrm{i}} / \mathrm{v}_{\mathrm{i}}=5 \times 10^{-4}$ and $\rho_{\mathrm{i}} / \mathrm{a}=3 \times 10^{-3}$, so the resulting inequality is typically satisfied.

In the next section, the tangential and radial drifts are evaluated for an imperfectly optimized stellarator along with transit and flux surface averages.

\section{Imperfectly optimized stellarator notation, drifts and averages}

To retain both the $1 / v$ and $\sqrt{v}$ regimes, the ion drift kinetic equation is employed (Hazeltine 1973; Simakov \& Catto 2005),

$$
\left(\mathrm{v}_{\|} \overrightarrow{\mathrm{b}}+\overrightarrow{\mathrm{v}}_{\mathrm{d}}\right) \cdot\left(\nabla \mathrm{f}_{1}+\frac{\mathrm{Zef}_{0}}{\mathrm{~T}} \nabla \phi\right)+\overrightarrow{\mathrm{v}}_{\mathrm{d}} \cdot \nabla \mathrm{f}_{0}=\mathrm{C}\left\{\mathrm{f}_{1}\right\}
$$

where $f_{1}$ is the perturbed distribution function, $C\left\{f_{1}\right\}$ is the linearized collision operator, $f_{0}$ is the Maxwellian

$$
\mathrm{f}_{0}=\mathrm{f}_{0}(\psi, \mathrm{E})=\mathrm{n}(\psi)\left[\frac{\mathrm{M}_{\mathrm{i}}}{2 \pi \mathrm{T}(\psi)}\right]^{3 / 2} \mathrm{e}^{-\mathrm{M}_{\mathrm{i}} \mathrm{v}^{2} / 2 \mathrm{~T}(\psi)}=\mathrm{n}(\psi)\left[\frac{\mathrm{M}_{\mathrm{i}}}{2 \pi \mathrm{T}(\psi)}\right]^{3 / 2} \mathrm{e}^{-\left[\mathrm{M}_{\mathrm{i}} \mathrm{E}-\mathrm{Ze} \Phi(\psi)\right] / \mathrm{T}(\psi)},
$$

and the drift velocity (ignoring the unimportant parallel velocity correction) is

$$
\overrightarrow{\mathrm{v}}_{\mathrm{d}}=\frac{\mathrm{c}}{\mathrm{B}^{2}} \overrightarrow{\mathrm{B}} \times \nabla \Phi+\frac{\mu}{\Omega} \overrightarrow{\mathrm{b}} \times \nabla \mathrm{B}+\frac{\mathrm{v}_{\|}^{2}}{\Omega} \overrightarrow{\mathrm{b}} \times(\overrightarrow{\mathrm{b}} \cdot \nabla \overrightarrow{\mathrm{b}}) \simeq \frac{\mathrm{v}_{\|}}{\Omega} \nabla \times\left(\mathrm{v}_{\|} \overrightarrow{\mathrm{b}}\right) .
$$

The kinetic equation will be solved in $\psi, \alpha=\zeta-q \vartheta, B, E=v^{2} / 2+\operatorname{Ze} \Phi(\psi) / M_{i}$, and $\mu=v_{\perp}^{2} / 2 B$ variables, with the lowest order electrostatic potential $\Phi$ taken as a flux function. The remaining spatial variables are $\vartheta$ and $\zeta$, the poloidal and toroidal angle variables, respectively, and the full electrostatic electric field is

$$
\overrightarrow{\mathrm{E}}=-\nabla[\Phi(\psi)+\phi(\psi, \vartheta, \zeta)]
$$

The magnetic field is $\vec{B}=B \vec{b}$ and the parallel velocity is $v_{\|}=\sigma v \xi$, with $\xi=\sqrt{1-\lambda B / B_{0}}$, pitch angle defined as $\lambda=2 \mu B_{0} / v^{2}, \sigma= \pm 1$, and $B_{0}$ a flux function $\left(B_{0}^{2}=\left\langle B^{2}\right\rangle\right)$. Here and throughout $\partial \mathrm{f}_{0} / \partial \psi$ is evaluated holding $\mathrm{E}$ fixed.

Using a Boozer (1981) representation for the magnetic field gives

$$
\overrightarrow{\mathrm{B}}=\mathrm{K}(\psi, \vartheta, \zeta) \nabla \psi+\mathrm{G}(\psi) \nabla \vartheta+\mathrm{I}(\psi) \nabla \zeta,
$$

with $\mathrm{K}$ periodic in $\vartheta$ and $\zeta$, and $\mathrm{RG} / \mathrm{rI} \sim \mathrm{B}_{\mathrm{p}} / \mathrm{B}$. The conventional stellarator poloidal and toroidal covariant flux functions ( $\mathrm{G}$ and $\mathrm{I}$ ) are interchanged in (3.5) to more closely conform to tokamak notation. The preceding and $\overrightarrow{\mathrm{B}}=\nabla \alpha \times \nabla \psi$ give $\mathrm{B}=(\mathrm{G}+\mathrm{qI}) \overrightarrow{\mathrm{b}} \cdot \nabla \vartheta$, as well as $\overrightarrow{\mathrm{B}} \cdot \nabla \alpha=0=\overrightarrow{\mathrm{B}} \cdot \nabla \psi$ and $\mathrm{q} \overrightarrow{\mathrm{B}} \cdot \nabla \vartheta=\overrightarrow{\mathrm{B}} \cdot \nabla \zeta$.

The well depth $\delta$ of the nearly omnigenous magnetic field is

$$
\delta=\left(\mathrm{B}_{\max }-\mathrm{B}_{\min }\right) /\left(\mathrm{B}_{\max }+\mathrm{B}_{\min }\right) .
$$

Here $B_{\max }$ and $B_{\min }$ denote the maximum and minimum field strength on the magnetic surface in question. As shown by Cary and Shasharina (1997a), in an omnigenous field these values are reached not in isolated points but along lines that close toroidally, poloidally or helically on the 
flux surface. Any departure from omnigeneity is assumed to result in smaller non-omnigenous magnetic field variation or weak ripple such tha

$$
\delta_{\text {no }}<<\delta \text {. }
$$

The non-omnigenous behavior can only introduce weak gradients comparable to those of the omnigeous portion of the magnetic field (Calvo et al. 2017) in order to avoid introducing any significant non-omnigenous magnetic wells. An additional, mild restriction on $\delta_{\text {no }}$ will be found in section 6. The omnigenous portion of the magnetic field is assumed to be $\mathrm{N}$ toroidal cells $(\mathrm{N}=$ 0 is quasiaxisymmetry) of well depth $\delta$, with $\bmod B$ contours closing on themselves after $M$ toroidal turns and $N$ poloidal turns. In $\psi, \alpha$, and $B$ variables $d \vartheta d \zeta=(\vec{b} \cdot \nabla \vartheta / \vec{b} \cdot \nabla B) d B d \alpha$. When performing flux surface averages it is convenient to be aware of the periodic variable

$$
\tilde{\alpha}=\alpha /(\mathrm{M}-\mathrm{qN})
$$

since $\tilde{\alpha} \rightarrow 2 \pi+\tilde{\alpha}=\tilde{\alpha}$ for $M$ toroidal circuits and $N$ poloidal circuits on a constant $B$ curve. As there are two points on either side of the $\mathrm{B}$ minimum, $\breve{\mathrm{B}}$, in each of the $\mathrm{N}$ cells, both sides must be summed or integrated over and are referred to as branches (Landreman \& Catto 2012).

In $\psi, \alpha, B$ variables the divergence of an arbitrary vector $\overrightarrow{\mathrm{A}}$ is

$$
\nabla \cdot \overrightarrow{\mathrm{A}}=\overrightarrow{\mathrm{B}} \cdot \nabla\left(\frac{\overrightarrow{\mathrm{A}} \cdot \nabla \mathrm{B}}{\overrightarrow{\mathrm{B}} \cdot \nabla \mathrm{B}}\right)+\overrightarrow{\mathrm{B}} \cdot \nabla \mathrm{B}\left[\frac{\partial}{\partial \alpha}\left(\frac{\overrightarrow{\mathrm{A}} \cdot \nabla \alpha}{\overrightarrow{\mathrm{B}} \cdot \nabla \mathrm{B}}\right)+\frac{\partial}{\partial \psi}\left(\frac{\overrightarrow{\mathrm{A}} \cdot \nabla \psi}{\overrightarrow{\mathrm{B}} \cdot \nabla \mathrm{B}}\right)\right] .
$$

The tangential $\overrightarrow{\mathrm{E}} \times \overrightarrow{\mathrm{B}}$ drift is then

$$
\overrightarrow{\mathrm{v}}_{\mathrm{d}} \cdot \nabla \alpha=\mathrm{v}_{\|} \overrightarrow{\mathrm{b}} \cdot \nabla\left(\frac{\mathrm{v}_{\|} \overrightarrow{\mathrm{B}} \cdot \nabla \alpha \times \nabla \mathrm{B}}{\Omega \overrightarrow{\mathrm{B}} \cdot \nabla \mathrm{B}}\right)+\mathrm{v}_{\|} \overrightarrow{\mathrm{b}} \cdot \nabla \mathrm{B} \frac{\partial}{\partial \psi}\left(\frac{\mathrm{v}_{\|} \mathrm{B}^{2}}{\Omega \overrightarrow{\mathrm{B}} \cdot \nabla \mathrm{B}}\right) \simeq \frac{\mathrm{Bv}_{\|}}{\Omega} \frac{\partial \mathrm{v}_{\|}}{\partial \psi} \simeq-\mathrm{c} \frac{\partial \Phi}{\partial \psi} \equiv-\omega(\psi),
$$

with $\omega$ positive for the ion root, and the radial drift is

$$
\overrightarrow{\mathrm{v}}_{\mathrm{d}} \cdot \nabla \psi=\mathrm{v}_{\|} \overrightarrow{\mathrm{b}} \cdot \nabla\left(\frac{\mathrm{v}_{\|} \overrightarrow{\mathrm{B}} \cdot \nabla \psi \times \nabla \mathrm{B}}{\Omega \overrightarrow{\mathrm{B}} \cdot \nabla \mathrm{B}}\right)-\frac{\mathrm{Bv}_{\|}^{2}}{\Omega} \overrightarrow{\mathrm{b}} \cdot \nabla \mathrm{B} \frac{\partial}{\partial \alpha}\left(\frac{1}{\overrightarrow{\mathrm{b}} \cdot \nabla \mathrm{B}}\right) .
$$

Using pressure balance to find $\vec{b} \times \nabla \psi \cdot(\nabla B-\vec{b} \cdot \nabla \vec{b})=0$ as in Helander \& Nührenberg (2009) and Landreman \& Catto (2012), gives the alternate radial drift form

$$
\overrightarrow{\mathrm{v}}_{\mathrm{d}} \cdot \nabla \psi=\frac{\overrightarrow{\mathrm{B}} \cdot \nabla \psi \times \nabla \mathrm{B}}{\overrightarrow{\mathrm{B}} \cdot \nabla \mathrm{B}} \mathrm{v}_{\|} \overrightarrow{\mathrm{b}} \cdot \nabla\left(\frac{\mathrm{v}_{\|}}{\Omega}\right)
$$

where for a quasisymmetric flux surface with $B_{q s}=B_{0}[1-\delta \cos (M \vartheta-N \xi)]$,

$$
\frac{\overrightarrow{\mathrm{B}} \cdot \nabla \psi \times \nabla \mathrm{B}}{\overrightarrow{\mathrm{B}} \cdot \nabla \mathrm{B}} \rightarrow \frac{\mathrm{MI}+\mathrm{NG}}{\mathrm{M}-\mathrm{qN}} \leqslant \mathrm{RB},
$$

with the tokamak and quasiaxisymmetric cases given by $\mathrm{N}=0$.

The transit average of an arbitray function $\mathrm{A}$ is defined variously as

$$
\overline{\mathrm{A}}=\frac{\oint_{\alpha} \mathrm{d} \ell \mathrm{A} / \mathrm{v}_{\|}}{\oint_{\alpha} \mathrm{d} \ell / \mathrm{v}_{\|}}=\frac{\oint_{\alpha} \mathrm{d} \tau \mathrm{A}}{\oint_{\alpha} \mathrm{d} \tau}=\frac{\oint_{\alpha} \mathrm{d} \vartheta \mathrm{A} / \mathrm{v}_{\|} \overrightarrow{\mathrm{b}} \cdot \nabla \vartheta}{\oint_{\alpha} \mathrm{d} \vartheta / \mathrm{v}_{\|} \overrightarrow{\mathrm{b}} \cdot \nabla \vartheta}=\frac{\sum \oint_{\alpha} \mathrm{dBA} / \mathrm{v}_{\|} \overrightarrow{\mathrm{b}} \cdot \nabla \mathrm{B}}{\sum \oint_{\alpha} \mathrm{dB} / \mathrm{v}_{\|} \overrightarrow{\mathrm{b}} \cdot \nabla \mathrm{B}},
$$

with $d \tau=d \ell / \mathrm{v}_{\|}=\mathrm{d} \vartheta / \mathrm{v}_{\|} \overrightarrow{\mathrm{b}} \cdot \nabla \vartheta=\mathrm{dB} / \mathrm{v}_{\|} \overrightarrow{\mathrm{b}} \cdot \nabla \mathrm{B}$. The integrals are over a full bounce for trapped particles and over a complete poloidal circuit for passing. A sum $\Sigma$ is inserted in integrals over all allowed B as a reminder that both "branches" or sides of the magnetic well must be integrated over (the two "branches" are on either side of the minimum $\breve{\mathrm{B}}$ in each identical stellarator cell). 
The subscript $\alpha$ on an integral means that it is to be held fixed while performing the integration. In a transit averaged description passing particles trace out flux surfaces, and it can take a passing particle many toroidal circuits in a non-omnigenous magnetic field to return to close to its starting field line point (Helander 2014). In particular, the passing flux surface average of $\mathrm{v}_{\|}$ can be viewed as

$$
\left\langle\mathrm{v}_{\|}\right\rangle_{\mathrm{p}}=\lim _{\mathrm{L} \rightarrow \infty}\left(\int_{0}^{\mathrm{L}} \mathrm{d} \ell \mathrm{v}_{\|} \mathrm{B}^{-1} / \int_{0}^{\mathrm{L}} \mathrm{d} \ell \mathrm{B}^{-1}\right)=\left(\oint \mathrm{d} \alpha \Sigma \oint \mathrm{dB}_{\|} / \overrightarrow{\mathrm{B}} \cdot \nabla \mathrm{B}\right) /(\oint \mathrm{d} \alpha \Sigma \oint \mathrm{dB} / \overrightarrow{\mathrm{B}} \cdot \nabla \mathrm{B}) .
$$

This expression is consistent with the usual definition of the flux surface average of any quantity A sampling the entire flux suface:

$$
\langle\mathrm{A}\rangle=\frac{\oint \mathrm{d} \zeta \oint \mathrm{d} \vartheta \mathrm{A} / \overrightarrow{\mathrm{B}} \cdot \nabla \vartheta}{\oint \mathrm{d} \xi \oint \mathrm{d} \vartheta / \overrightarrow{\mathrm{B}} \cdot \nabla \vartheta}=\frac{\oint \mathrm{d} \zeta \oint \mathrm{d} \vartheta \mathrm{A} / \mathrm{B}^{2}}{\oint \mathrm{d} \xi \oint \mathrm{d} \vartheta / \mathrm{B}^{2}}=\frac{\oint \mathrm{d} \alpha \Sigma \oint \mathrm{dB} \mathrm{A} / \overrightarrow{\mathrm{B}} \cdot \nabla \mathrm{B}}{\oint \mathrm{d} \alpha \Sigma \oint \mathrm{dB} / \overrightarrow{\mathrm{B}} \cdot \nabla \mathrm{B}}
$$

The relation between transit and flux surface averages for passing and $\mathrm{A}(\psi, \alpha, \mathrm{B}, \mathrm{v}, \lambda)$ is

$$
\left.\overline{\mathrm{A}}\right|_{\mathrm{p}}=\left\langle\mathrm{BA} / \mathrm{v}_{\|}\right\rangle_{\mathrm{p}} /\left\langle\mathrm{B} / \mathrm{v}_{\|}\right\rangle_{\mathrm{p}} \text {. }
$$

A few useful properties associated with a MHD equilibrium are briefly discussed next.

\section{MHD equilibrium properties and radial drift}

Some useful relations follow from conditions associated with force balance and ambipolarity. Force balance $(\vec{J} \times \vec{B}=c \nabla p)$ and charge conservation $(\nabla \cdot \vec{J}=0)$ require there be no radial current $(\vec{J} \cdot \nabla \psi=0)$, where $\mathrm{p}=\mathrm{p}_{\mathrm{i}}+\mathrm{p}_{\mathrm{e}}$ is the total pressure and the current density is $\overrightarrow{\mathrm{J}}=\mathrm{cB}^{-2} \overrightarrow{\mathrm{B}} \times \nabla \mathrm{p}(\psi)+\mathrm{J}_{\|} \overrightarrow{\mathrm{b}}$. Ambipolarity, $\overrightarrow{\mathrm{J}} \cdot \nabla \psi=0$, requires $\nabla \psi \cdot \nabla \times \overrightarrow{\mathrm{B}}=0=\nabla \cdot(\overrightarrow{\mathrm{B}} \times \nabla \psi)$, giving

$$
\overrightarrow{\mathrm{B}} \cdot \nabla\left(\frac{\overrightarrow{\mathrm{B}} \cdot \nabla \psi \times \nabla \mathrm{B}}{\overrightarrow{\mathrm{B}} \cdot \nabla \mathrm{B}}\right)=\overrightarrow{\mathrm{B}} \cdot \nabla \mathrm{B} \frac{\partial}{\partial \alpha}\left(\frac{\mathrm{B}^{2}}{\overrightarrow{\mathrm{B}} \cdot \nabla \mathrm{B}}\right),
$$

Integrating along a field line (fixed $\alpha$ ) on the flux surface traced out by the field line gives

$$
\frac{\overrightarrow{\mathrm{B}} \cdot \nabla \psi \times \nabla \mathrm{B}}{\overrightarrow{\mathrm{B}} \cdot \nabla \mathrm{B}}-\frac{\partial}{\partial \alpha} \int_{\mathrm{B}_{0}}^{\mathrm{B}} \frac{\mathrm{dB}^{\prime} \mathrm{B}^{\prime}}{\overrightarrow{\mathrm{b}}^{\prime} \cdot \nabla \mathrm{B}^{\prime}}=\mathrm{X}(\psi)=\left\langle\frac{\overrightarrow{\mathrm{B}} \cdot \nabla \psi \times \nabla \mathrm{B}}{\overrightarrow{\mathrm{B}} \cdot \nabla \mathrm{B}}\right\rangle-\left\langle\frac{\partial}{\partial \alpha_{\mathrm{B}_{0}}} \int_{\mathrm{b}^{\prime} \cdot \nabla \mathrm{B}^{\prime}}^{\mathrm{B}} \frac{\mathrm{dB}^{\prime} \mathrm{B}^{\prime}}{\overrightarrow{\mathrm{b}}^{\prime}},\right.
$$

where $B_{0}^{2}=\left\langle B^{2}\right\rangle$ is chosen so that the lower limit will satisfy $\vec{b} \cdot \nabla B_{0}=0$ as desired. The $\alpha$ derivatives are always at fixed $\mathrm{B}$ unless otherwise noted, and $\partial \mathrm{B}_{0} / \partial \alpha=0$. In an omnigenous system the lower limit $B_{0}$ of the integral over $B$ can be replaced by another $\alpha$ independent value such as $\widehat{B}=B_{\max }$ or $\breve{B}=B_{\min }$. The last equality in (4.2), follows upon flux surface averaging.

As a result, ambipolarity always requires

$$
\frac{\overrightarrow{\mathrm{B}} \cdot \nabla \psi \times \nabla \mathrm{B}}{\overrightarrow{\mathrm{B}} \cdot \nabla \mathrm{B}}=\left\langle\frac{\overrightarrow{\mathrm{B}} \cdot \nabla \psi \times \nabla \mathrm{B}}{\overrightarrow{\mathrm{B}} \cdot \nabla \mathrm{B}}\right\rangle+\frac{\partial \mathrm{F}_{0}}{\partial \alpha}-\left\langle\frac{\partial \mathrm{F}_{0}}{\partial \alpha}\right\rangle,
$$

where the integrals over $\mathrm{B}$ are at fixed $\alpha$, and $\mathrm{F}_{0}$ is defined as

$$
\mathrm{F}_{0}=\int_{\mathrm{B}_{0}}^{\mathrm{B}} \frac{\mathrm{dB}^{\prime} \mathrm{B}^{\prime}}{\overrightarrow{\mathrm{b}}^{\prime} \cdot \nabla \mathrm{B}^{\prime}}
$$

Integrating over both branches on either side of the the single minimum $\breve{\mathrm{B}}=\mathrm{B}_{\text {min }}$ gives $\left\langle\partial \mathrm{F}_{0} / \partial \alpha\right\rangle=0$ according to (1.3). 
In Eqs. (4.2) and (4.4), the integrals are taken at fixed $\psi$ and $\alpha$ (therefore following a field line) starting from a point where $\mathrm{B}^{\prime}=\mathrm{B}_{0}$ and proceeding along the field to the point where $\mathrm{B}^{\prime}=\mathrm{B}$. As already mentioned, in an omnigenous field, all level curves $\mathrm{B}=\mathrm{B}_{0}$ wrap around the torus poloidally, toroidally, or helically (Cary \& Shasharina 1997a), so that it is indeed possible to find a point where $\mathrm{B}=\mathrm{B}_{0}$ on each field line within one period of the device. The field is allowed to be non-omnigenous, but only slightly so, in order that it still possesses this property. Otherwise it would be difficult to ascribe any precise meaning to these integrals.

Force balance and charge conservation require a geometrical function $U$ proportional to $\mathrm{J}_{\|}$to exist satisfying

$$
\overrightarrow{\mathrm{B}} \cdot \nabla(\mathrm{U} / \mathrm{B})=\overrightarrow{\mathrm{B}} \cdot \nabla \psi \times \nabla \mathrm{B}^{-2}=\nabla \cdot\left(\mathrm{B}^{-2} \overrightarrow{\mathrm{B}} \times \nabla \psi\right) .
$$

Rewriting using the divergence in $\psi, \alpha$ and $\mathrm{B}$ variables,

$$
\overrightarrow{\mathrm{B}} \cdot \nabla\left(\frac{\mathrm{U}}{\mathrm{B}}-\frac{\overrightarrow{\mathrm{B}} \cdot \nabla \psi \times \nabla \mathrm{B}}{\mathrm{B}^{2} \overrightarrow{\mathrm{B}} \cdot \nabla \mathrm{B}}\right)=-\overrightarrow{\mathrm{b}} \cdot \nabla \mathrm{B} \frac{\partial}{\partial \alpha}\left(\frac{1}{\overrightarrow{\mathrm{b}} \cdot \nabla \mathrm{B}}\right) .
$$

Using the same procedure to integrate over the flux surface traced out by a field line leads to

$$
\mathrm{BU}-\frac{\overrightarrow{\mathrm{B}} \cdot \nabla \psi \times \nabla \mathrm{B}}{\overrightarrow{\mathrm{B}} \cdot \nabla \mathrm{B}}+\mathrm{B}^{2} \frac{\partial}{\partial \alpha_{\mathrm{B}_{0}}} \int_{\overrightarrow{\mathrm{B}}^{\prime} \cdot \nabla \mathrm{B}^{\prime}}^{\mathrm{B}}=\mathrm{dB}(\psi) \mathrm{B}^{2} .
$$

Flux surface averaging gives $\mathrm{Y}$

$$
\mathrm{Y}(\psi)\left\langle\mathrm{B}^{2}\right\rangle=\langle\mathrm{BU}\rangle-\left\langle\frac{\overrightarrow{\mathrm{B}} \cdot \nabla \psi \times \nabla \mathrm{B}}{\overrightarrow{\mathrm{B}} \cdot \nabla \mathrm{B}}\right\rangle+\left\langle\mathrm{B}^{2} \frac{\partial}{\partial \alpha} \int_{\mathrm{B}_{0}}^{\mathrm{B}} \frac{\mathrm{dB}^{\prime}}{\overrightarrow{\mathrm{B}}^{\prime} \cdot \nabla \mathrm{B}^{\prime}}\right\rangle .
$$

Eliminating Y yields

$$
\frac{\mathrm{U}}{\mathrm{B}}-\frac{\langle\mathrm{B} U\rangle}{\left\langle\mathrm{B}^{2}\right\rangle}=\left(\frac{\overrightarrow{\mathrm{B}} \cdot \nabla \psi \times \nabla \mathrm{B}}{\mathrm{B}^{2} \overrightarrow{\mathrm{B}} \cdot \nabla \mathrm{B}}-\frac{\partial}{\partial \alpha_{\mathrm{B}_{0}}} \int_{\mathrm{B}^{\prime} \cdot \nabla \mathrm{B}^{\prime}}^{\mathrm{B}} \frac{\mathrm{dB}^{\prime}}{\left.\overrightarrow{\mathrm{B}}^{\prime}\right\rangle}\left(\left\langle\frac{1}{\langle\overrightarrow{\mathrm{B}} \cdot \nabla \psi \times \nabla \mathrm{B}}\right\rangle-\left\langle\mathrm{B}^{2} \frac{\partial}{\partial \alpha_{\mathrm{B}_{0}}} \int_{\mathrm{B}^{\prime} \cdot \nabla \mathrm{B}^{\prime}}^{\mathrm{B}} \frac{\mathrm{dB}^{\prime}}{\overrightarrow{\mathrm{B}}^{\prime}}\right) .\right.\right.
$$

Terms in $\mathrm{U} / \mathrm{B}$ that are only flux functions do not contribute to $\overrightarrow{\mathrm{B}} \cdot \nabla(\mathrm{U} / \mathrm{B})$. Therefore, picking

$$
\langle\mathrm{BU}\rangle=\left\langle\frac{\overrightarrow{\mathrm{B}} \cdot \nabla \psi \times \nabla \mathrm{B}}{\overrightarrow{\mathrm{B}} \cdot \nabla \mathrm{B}}\right\rangle-\left\langle\mathrm{B}^{2} \frac{\partial}{\partial \alpha_{\mathrm{B}_{0}}} \int_{\mathrm{B}^{\prime} \cdot \nabla \mathrm{B}^{\prime}}^{\mathrm{B}} \frac{\mathrm{dB}^{\prime}}{\overrightarrow{\mathrm{B}}^{\prime}},\right.
$$

where the last term vanishes for an omnigenous flux surface due to (1.3), leaves

$$
\mathrm{BU}=\frac{\overrightarrow{\mathrm{B}} \cdot \nabla \psi \times \nabla \mathrm{B}}{\overrightarrow{\mathrm{B}} \cdot \nabla \mathrm{B}}-\mathrm{B}^{2} \frac{\partial}{\partial \alpha} \int_{\mathrm{B}_{0}}^{\mathrm{B}} \frac{\mathrm{dB}^{\prime}}{\overrightarrow{\mathrm{B}^{\prime} \cdot \nabla \mathrm{B}^{\prime}}}=\left\langle\frac{\overrightarrow{\mathrm{B}} \cdot \nabla \psi \times \nabla \mathrm{B}}{\overrightarrow{\mathrm{B}} \cdot \nabla \mathrm{B}}\right\rangle-\left\langle\frac{\partial \mathrm{F}_{0}}{\partial \alpha}\right\rangle+\frac{\partial}{\partial \alpha} \int_{\mathrm{B}_{0}}^{\mathrm{B}} \frac{\mathrm{dB}^{\prime}\left(\mathrm{B}^{\prime 2}-\mathrm{B}^{2}\right)}{\overrightarrow{\mathrm{B}}^{\prime} \cdot \nabla \mathrm{B}^{\prime}},
$$

and the last form follows by using ambipolarity. As a result, BU is a flux function for a quasisymmetric flux surface, or when summed over both branches on an omnigenous surface. Otherwise, $\mathrm{BU}$ is nearly a flux function when $\mathrm{B}^{2}>>\widehat{\mathrm{B}}^{2}-\breve{\mathrm{B}}^{2}$, where $\widehat{\mathrm{B}}=\mathrm{B}_{\max }$ and $\breve{\mathrm{B}}=\mathrm{B}_{\min }$.

The next section formulates the drift kinetic equation in a particularly convenient form.

\section{Drift kinetics for imperfectly optimized stellarators}

To find the modifications to the Maxwellian it is convenient to let

$$
\mathrm{f}_{1}=\mathrm{h}(\overrightarrow{\mathrm{r}}, \overrightarrow{\mathrm{v}})-\mathrm{Ze}_{\mathrm{f}} \mathrm{f}_{0} / \mathrm{T},
$$

then using form (3.12) for the radial drift, h satisfies 


$$
\mathrm{v}_{\|} \overrightarrow{\mathrm{b}} \cdot \nabla \mathrm{h}+\overrightarrow{\mathrm{v}}_{\mathrm{d}} \cdot \nabla \mathrm{h}-\mathrm{C}\{\mathrm{h}\}=-\frac{\overrightarrow{\mathrm{B}} \cdot \nabla \psi \times \nabla \mathrm{B}}{\overrightarrow{\mathrm{B}} \cdot \nabla \mathrm{B}} \frac{\partial \mathrm{f}_{0}}{\partial \psi} \mathrm{v}_{\|} \overrightarrow{\mathrm{b}} \cdot \nabla\left(\frac{\mathrm{v}_{\|}}{\Omega}\right) .
$$

where radial nonlocality and tangential drift are included in $\overrightarrow{\mathrm{v}}_{\mathrm{d}} \cdot \nabla \mathrm{h}_{\mathrm{p}}$ via $\overrightarrow{\mathrm{v}}_{\mathrm{d}} \cdot \nabla \psi \partial \mathrm{h}_{\mathrm{p}} / \partial \psi$ and $\overrightarrow{\mathrm{v}}_{\mathrm{d}} \cdot \nabla \alpha \partial \mathrm{h}_{\mathrm{p}} / \partial \alpha$, respectively. In writing (5.2), a Ze $\phi \overrightarrow{\mathrm{v}}_{\mathrm{d}} \cdot \nabla\left(\mathrm{f}_{0} / \mathrm{T}\right)$ correction to $\overrightarrow{\mathrm{v}}_{\mathrm{d}} \cdot \nabla \mathrm{f}_{0}$ is ignored as small. For radial transport calculations and in many simulations the local approximation $\overrightarrow{\mathrm{v}}_{\mathrm{d}} \cdot \nabla \mathrm{h} \rightarrow-\omega \partial \mathrm{h} / \partial \alpha$ is employed. However, it will be shown in the next section that this replacement gives rise to spurious effects that will be considered in section 8. Consequently, the full drift is retained until section 8, although in making estimates only the $\overrightarrow{\mathrm{E}} \times \overrightarrow{\mathrm{B}}$ drift is retained.

Rewriting the preceding using (4.1) gives the form

$$
\mathrm{v}_{\|} \overrightarrow{\mathrm{b}} \cdot \nabla\left(\mathrm{h}+\frac{\mathrm{v}_{\|} \overrightarrow{\mathrm{B}} \cdot \nabla \psi \times \nabla \mathrm{B}}{\Omega \overrightarrow{\mathrm{B}} \cdot \nabla \mathrm{B}} \frac{\partial \mathrm{f}_{0}}{\partial \psi}\right)+\overrightarrow{\mathrm{v}}_{\mathrm{d}} \cdot \nabla \mathrm{h}-\mathrm{C}\{\mathrm{h}\}=\frac{\mathrm{B} \partial \mathrm{f}_{0}}{\Omega \partial \psi} \mathrm{v}_{\|} \overrightarrow{\mathrm{b}} \cdot \nabla \mathrm{B} \frac{\partial}{\partial \alpha}\left(\frac{\mathrm{v}_{\|}}{\overrightarrow{\mathrm{b}} \cdot \nabla \mathrm{B}}\right),
$$

and, thereby, the particularly convenient form

where

$$
\mathrm{C}\{\mathrm{h}\}-\overrightarrow{\mathrm{v}}_{\mathrm{d}} \cdot \nabla \mathrm{h}=\mathrm{v}_{\|} \overrightarrow{\mathrm{b}} \cdot \nabla \mathrm{H}=\mathrm{v}_{\|} \overrightarrow{\mathrm{b}} \cdot \nabla \mathrm{h}+\mathrm{v}_{\|} \overrightarrow{\mathrm{b}} \cdot \nabla \Delta \frac{\partial \mathrm{f}_{0}}{\partial \psi}=\mathrm{v}_{\|} \overrightarrow{\mathrm{b}} \cdot \nabla \mathrm{h}+\overrightarrow{\mathrm{v}}_{\mathrm{d}} \cdot \nabla \psi \frac{\partial \mathrm{f}_{0}}{\partial \psi},
$$

$$
\begin{gathered}
\mathrm{H} \equiv \mathrm{h}+\Delta \partial \mathrm{f}_{0} / \partial \psi, \\
\Delta \equiv \frac{\mathrm{v}_{\|} \overrightarrow{\mathrm{B}} \cdot \nabla \psi \times \nabla \mathrm{B}}{\Omega \overrightarrow{\mathrm{B}} \cdot \nabla \mathrm{B}}-\frac{\partial \tilde{\mathrm{J}}}{\partial \alpha}=\frac{\mathrm{v}_{\|}}{\Omega}\left(\left\langle\frac{\overrightarrow{\mathrm{B}} \cdot \nabla \psi \times \nabla \mathrm{B}}{\overrightarrow{\mathrm{B}} \cdot \nabla \mathrm{B}}\right\rangle-\left\langle\frac{\partial \mathrm{F}_{0}}{\partial \alpha}\right\rangle+\frac{\partial \mathrm{F}_{0}}{\partial \alpha}\right)-\frac{\partial \tilde{\mathrm{J}}}{\partial \alpha},
\end{gathered}
$$

and

$$
\tilde{\mathrm{J}} \equiv \frac{\mathrm{Mc}}{\mathrm{Ze}} \int_{\overline{\mathrm{B}}}^{\mathrm{B}} \frac{\mathrm{dB}^{\prime} \mathrm{v}_{\|}^{\prime}}{\overrightarrow{\mathrm{b}}^{\prime} \cdot \nabla \mathrm{B}^{\prime}},
$$

with $v_{\|}^{\prime}=v_{\|}\left(B^{\prime}, v, \lambda\right)$. The function $\tilde{J}$ is related to, but not the same as, the second adiabatic invariant (1.4), for which $\partial \mathrm{J} / \partial \alpha=0$ on an omnigenous flux surface. The lower limit $\overline{\mathrm{B}}$ in $\tilde{\mathrm{J}}$ remains unspecified except that it must satisfy $\partial \overline{\mathrm{B}} / \partial \alpha=0$ to allow

$$
\left(\int_{\overline{\mathrm{B}}}^{\mathrm{B}} \mathrm{d \textrm {B } ^ { \prime }} \mathrm{v}_{\|} \frac{\partial}{\partial \alpha}\left(\frac{1}{\overrightarrow{\mathrm{b}}^{\prime} \cdot \nabla \mathrm{B}^{\prime}}\right)=\frac{\partial}{\partial \alpha}\left(\int_{\overline{\mathrm{B}}}^{\mathrm{B}} \frac{\mathrm{d \textrm {B } ^ { \prime }} \mathrm{v}_{\|}^{\prime} \cdot \nabla \mathrm{B}^{\prime}}{\overrightarrow{\mathrm{b}}^{\prime}}\right) .\right.
$$

The lack of precise omnigeneity means that $\overline{v_{\|} \vec{b} \cdot \nabla \Delta}$ no longer quite vanishes,

$$
\overline{\mathrm{v}_{\|} \overrightarrow{\mathrm{b}} \cdot \nabla \Delta}=\overline{\mathrm{v}_{\|} \overrightarrow{\mathrm{b}} \cdot \nabla\left(\frac{\mathrm{v}_{\|} \partial \mathrm{F}_{0}}{\Omega \partial \alpha}-\frac{\partial \tilde{\mathrm{J}}}{\partial \alpha}\right)}=\overline{\mathrm{v}_{\|} \overrightarrow{\mathrm{b}} \cdot \nabla \mathrm{B}\left\{\frac{\partial}{\partial \alpha}\left[\frac{\partial}{\partial \mathrm{B}}\left(\frac{\mathrm{v}_{\|}}{\Omega} \mathrm{F}_{0}-\tilde{\mathrm{J}}\right)\right]\right\}}=\overline{\frac{\partial \mathrm{F}_{0}}{\partial \alpha} \mathrm{v}_{\|} \overrightarrow{\mathrm{b}} \cdot \nabla\left(\frac{\mathrm{v}_{\|}}{\Omega}\right)} \neq 0,
$$

as $\alpha$ dependence causes $\Sigma \partial \mathrm{F}_{0} / \partial \alpha \neq 0$ and $\Sigma \partial \tilde{\mathrm{J}} / \partial \alpha \neq 0$ when summed over both branches.

The kinetic equation (5.4) agrees with the omnigenous form obtained from (3.12) by Helander \& Nührenberg (2009) and Landreman \& Catto (2012). They also write

$$
\overrightarrow{\mathrm{v}}_{\mathrm{d}} \cdot \nabla \psi=\mathrm{v}_{\|} \overrightarrow{\mathrm{b}} \cdot \nabla \Delta \text {, }
$$

but make the replacement

$$
\Delta \rightarrow \Delta_{\text {om }} \equiv \frac{\mathrm{v}_{\mathrm{II}}}{\Omega}\left\langle\frac{\overrightarrow{\mathrm{B}} \cdot \nabla \psi \times \nabla \mathrm{B}}{\overrightarrow{\mathrm{B}} \cdot \nabla \mathrm{B}}\right\rangle^{\mathrm{B}}+\int_{\overline{\mathrm{B}}}^{\mathrm{B}} \mathrm{dB}^{\prime} \frac{\partial \mathrm{F}_{0}^{\prime} \partial}{\partial \alpha \partial \mathrm{B}^{\prime}}\left(\frac{\mathrm{v}_{\mathrm{II}}^{\prime}}{\Omega^{\prime}}\right),
$$

where $\overline{\mathrm{B}}=\widehat{\mathrm{B}}=\mathrm{B}_{\max }$ when $\lambda<\mathrm{B}_{0} / \widehat{\mathrm{B}}$, and $\overline{\mathrm{B}}=\mathrm{B}_{0} / \lambda$ otherwise, as their choice of the lower limit. To see the results here are consistent with their omnigenous results, integrate by parts to obtain 


$$
\frac{\mathrm{v}_{\|}}{\Omega} \frac{\partial \mathrm{F}_{0}}{\partial \alpha}-\frac{\partial \tilde{J}}{\partial \alpha}=\int_{\overline{\mathrm{B}}}^{\mathrm{B}} \mathrm{dB}^{\prime} \frac{\partial \mathrm{F}_{0}^{\prime} \partial}{\partial \alpha \partial \mathrm{B}^{\prime}}\left(\frac{\mathrm{v}_{\|}^{\prime}}{\Omega^{\prime}}\right)=-\frac{\mathrm{Bv}^{\prime}}{2 \Omega} \int_{\overline{\mathrm{B}}}^{\mathrm{B}} \frac{\mathrm{dB}^{\prime} \partial \mathrm{F}_{0}^{\prime}\left(2-\lambda \mathrm{B}^{\prime} / \mathrm{B}_{0}\right)}{\mathrm{B}^{\prime 2} \partial \alpha \sqrt{1-\lambda \mathrm{B}^{\prime} / \mathrm{B}_{0}}},
$$

where $v_{\|}^{\prime}=v_{\|}\left(B^{\prime}=\bar{B}, v, \lambda\right)=0$ is needed.

The omnigenous limit is recovered only if the lower limits used by Helander \& Nührenberg (2009) and Landreman \& Catto (2012) can be employed. The alternate form given here only reduces to their form (5.10) and (5.11) for an omnigenous flux surface. More generally, $\Delta$ must satisfy (5.6) with the lower limit $\overline{\mathrm{B}}$ of $\tilde{\mathbf{J}}$ equal to $\widehat{\mathrm{B}}=\mathrm{B}_{\max }$ for the passing $\left(\lambda<\mathrm{B}_{0} / \widehat{\mathrm{B}}\right)$ and $\mathrm{B}_{0} / \lambda$ for the trapped $\left(\mathrm{B}_{0} / \mathrm{B}_{\min }=\mathrm{B}_{0} / \breve{\mathrm{B}}>\lambda>\mathrm{B}_{0} / \widehat{\mathrm{B}}\right)$ with a negligible population of transitional particles that spend time trapped in multiple wells.

In the next section the corrections to the Maxwellian are evaluated for a non-optimized stellarator by assuming that the population of transitional particles is small.

\section{Solution for a weak departure from omnigeneity}

The drift kinetic equation will be solved separately for the trapped $(\mathrm{t})$ and passing $(\mathrm{p})$, using subscripts $t$ and $\mathrm{p}$ as necessary for clarity. A solution to the passing kinetic equation (5.4) is obtained by recalling (5.4) and (5.5), writing

$$
\mathrm{H}_{\mathrm{p}}=\overline{\mathrm{H}}_{\mathrm{p}}+\tilde{\mathrm{H}}_{\mathrm{p}} \text {, }
$$

where $\overline{\mathrm{H}}_{\mathrm{p}} \gg \tilde{\mathrm{H}}_{\mathrm{p}}$. Assuming streaming dominates, then to lowest order

where

$$
\mathrm{v}_{\|} \overrightarrow{\mathrm{b}} \cdot \nabla \overline{\mathrm{H}}_{\mathrm{p}}=0
$$

$$
\overline{\mathrm{H}}_{\mathrm{p}}=\overline{\mathrm{H}}_{\mathrm{p}}(\psi, \mathrm{v}, \lambda, \sigma)
$$

To next order

$$
\mathrm{v}_{\|} \overrightarrow{\mathrm{b}} \cdot \nabla \tilde{H}_{\mathrm{p}}=\mathrm{C}\left\{\mathrm{h}_{\mathrm{p}}\right\}-\overrightarrow{\mathrm{v}}_{\mathrm{d}} \cdot \nabla \mathrm{h}_{\mathrm{p}} .
$$

Upon transit averaging and using (3.19) the right side vanishes

$$
\begin{aligned}
& \left\langle\frac{\mathrm{B}}{\mathrm{V}_{\|}} \mathrm{C}\left\{\mathrm{h}_{\mathrm{p}}\right\}\right\rangle_{\mathrm{p}}=\left\langle\frac{\mathrm{B}}{\mathrm{V}_{\|}} \overrightarrow{\mathrm{V}}_{\mathrm{d}} \cdot \nabla \mathrm{h}_{\mathrm{p}}\right\rangle_{\mathrm{p}}=\left\langle\nabla \cdot\left(\frac{\mathrm{B}}{\mathrm{V}_{\|}} \overrightarrow{\mathrm{V}}_{\mathrm{d}} \mathrm{h}_{\mathrm{p}}\right)\right\rangle=\frac{1}{\mathrm{~V}^{\prime}} \frac{\partial}{\partial \psi} \mathrm{V}^{\prime}\left[\left\langle\left(\overline{\mathrm{H}}_{\mathrm{p}}-\Delta_{\mathrm{p}} \frac{\partial \mathrm{f}_{0}}{\partial \psi}\right) \frac{\mathrm{B}}{\mathrm{V}_{\|}} \overrightarrow{\mathrm{v}}_{\mathrm{d}} \cdot \nabla \psi\right\rangle\right. \\
& =\frac{1}{\mathrm{~V}^{\prime}} \frac{\partial}{\partial \psi} \mathrm{V}^{\prime}\left[\left\langle\left(\overline{\mathrm{H}}_{\mathrm{p}}-\Delta_{\mathrm{p}} \frac{\partial \mathrm{f}_{0}}{\partial \psi}\right) \overrightarrow{\mathrm{B}} \cdot \nabla \Delta_{\mathrm{p}}\right\rangle=\frac{1}{\mathrm{~V}^{\prime}} \frac{\partial}{\partial \psi} \mathrm{V}^{\prime}\left[\overline{\mathrm{H}}_{\mathrm{p}}\left\langle\overrightarrow{\mathrm{B}} \cdot \nabla \Delta_{\mathrm{p}}\right\rangle-\frac{\partial \mathrm{f}_{0}}{\partial \psi}\left\langle\overrightarrow{\mathrm{B}} \cdot \nabla\left(\frac{\Delta_{\mathrm{p}}^{2}}{2}\right)\right\rangle\right]=0\right.
\end{aligned},
$$

where $\overrightarrow{\mathrm{v}}_{\mathrm{d}} \cdot \nabla \psi=\mathrm{v}_{\|} \overrightarrow{\mathrm{b}} \cdot \nabla \Delta_{\mathrm{p}}$ and $\mathrm{V}^{\prime}=\oint \mathrm{d} \zeta \oint \mathrm{d} \vartheta / \mathrm{B}^{2}$ are employed.

The preceding careful drift kinetic treatment retaining the full nonlocal behavior of the drift departure from a flux surface results in the constraint

$$
\left\langle\frac{\mathrm{B}}{\mathrm{v}_{\|}} \mathrm{C}\left\{\mathrm{h}_{\mathrm{p}}\right\}\right\rangle_{\mathrm{p}}=0 .
$$

However, most simulations retain effects associated with solving for $\mathrm{h}$ when the approximation

$$
\overrightarrow{\mathrm{v}}_{\mathrm{d}} \cdot \nabla \mathrm{h} \rightarrow-\omega(\psi) \partial \mathrm{h} / \partial \alpha \simeq \omega(\psi)(\partial \Delta / \partial \alpha) \partial \mathrm{f}_{0} / \partial \psi,
$$

is employed. When used in (6.5), it gives the specious constraint

$$
\left\langle\frac{\mathrm{B}}{\mathrm{v}_{\|}} \mathrm{C}\left\{\mathrm{h}_{\mathrm{p}}\right\}\right\rangle \rightarrow \omega \frac{\partial \mathrm{f}_{0}}{\partial \psi}\left\langle\frac{\mathrm{B} \partial \Delta_{\mathrm{p}}}{\mathrm{v}_{\|} \partial \alpha}\right\rangle=\omega \frac{\partial \mathrm{f}_{0}}{\partial \psi}\left[\frac{\mathrm{B}}{\Omega}\left\langle\frac{\partial^{2} \mathrm{~F}_{0}}{\partial \alpha^{2}}\right\rangle-\left\langle\frac{\mathrm{B} \partial^{2} \tilde{\mathrm{J}}}{\mathrm{V}_{\|} \partial \alpha^{2}}\right\rangle\right]_{\mathrm{p}} \neq 0 .
$$


As the departure from omnigeneity is assumed weak $\left(\delta_{\text {no }}<\delta\right.$ ) and the tangential drift $\omega$ and collision terms are small, it is tempting to perform the flux surface averages by assuming the flux surface is omnigenous to lowest order to make $\left\langle\mathrm{Bv}_{\|}^{-1} \partial \Delta_{\mathrm{p}} / \partial \alpha\right\rangle$ vanish. However, as the approximation (6.7) has been employed in most numerical simulations, integrating the right side of (6.8) by parts in $\alpha$, results in B integrals of $\alpha$ derivatives of the $\vec{b} \cdot \nabla B$ from the flux surface averages. Therefore, any departure from omnigeneity gives $\left\langle\mathrm{Bv}_{\|}^{-1} \partial \Delta_{\mathrm{p}} / \partial \alpha\right\rangle \neq 0$, which will contribute at very low collisionalities and lead to questionable behavior in the results for the flow and bootstrap current as discussed further in section 8 .

Returning to the passing constraint (6.6) leads to the lowest order relation

$$
\mathrm{h}_{\mathrm{p}}=\overline{\mathrm{H}}_{\mathrm{p}}-\Delta_{\mathrm{p}} \partial \mathrm{f}_{0} / \partial \psi \text {. }
$$

Using $\overrightarrow{\mathrm{B}} \cdot \nabla \psi \times \nabla \mathrm{B} / \overrightarrow{\mathrm{B}} \cdot \nabla \mathrm{B} \sim \mathrm{BU} \lesssim \mathrm{RB}$, the transit averaged constraint $\left\langle\mathrm{Bv}_{\|}^{-1} \mathrm{C}\left\{\mathrm{h}_{\mathrm{p}}\right\}\right\rangle_{\mathrm{p}}=0$ suggests that for the ions

$$
\frac{\mathrm{h}_{\mathrm{p}}}{\mathrm{f}_{0}} \sim \frac{\overline{\mathrm{H}}_{\mathrm{p}}}{\mathrm{f}_{0}} \sim \frac{\Delta_{\mathrm{p}}}{\mathrm{RB}_{\mathrm{p}} \mathrm{a}} \lesssim \frac{\rho_{\mathrm{pi}}}{\mathrm{a}},
$$

while the equation non-averaged equation (6.4) with $\vec{v}_{d} \cdot \nabla h_{p} \rightarrow-\omega \partial h_{p} / \partial \alpha$ gives

$$
\frac{\tilde{\mathrm{H}}_{\mathrm{p}}}{\mathrm{f}_{0}} \sim \frac{\left(\omega+v_{\mathrm{i}}\right) \mathrm{R}}{\mathrm{v}_{\mathrm{i}}} \frac{\overline{\mathrm{H}}_{\mathrm{p}}}{\mathrm{f}_{0}}<\frac{\left(\omega+v_{\mathrm{i}}\right) \mathrm{R} \rho_{\mathrm{pi}}}{\mathrm{v}_{\mathrm{i}} \mathrm{a}},
$$

where $\left(\omega+v_{i}\right) R<v_{i}$. Here and elsewhere, $\rho_{p i}=\rho_{i} B / B_{p}$, and $\rho_{i}$ the ion gyroradius and $B_{p}$ the poloidal magnetic field. As $\overline{\mathrm{H}}_{\mathrm{p}}$ and $\Delta_{\mathrm{p}}$ are odd in $\mathrm{v}_{\|}$in (6.6), $\tilde{\mathrm{H}}_{\mathrm{p}}$ from (6.4) will be even in $\mathrm{v}_{\|}$.

Here and in the next section, the correct constraint equation (6.6) is solved by considering

$$
0=\left\langle\frac{\mathrm{B}}{\mathrm{V}_{\|}} \mathrm{C}\left\{\overline{\mathrm{H}}_{\mathrm{p}}-\frac{\mathrm{v}_{\|}}{\Omega}\left\langle\frac{\overrightarrow{\mathrm{B}} \cdot \nabla \psi \times \nabla \mathrm{B}}{\overrightarrow{\mathrm{B}} \cdot \nabla \mathrm{B}}\right\rangle \frac{\partial \mathrm{f}_{0}}{\partial \psi}\right\}\right\rangle=\left\langle\frac{\mathrm{B}}{\mathrm{V}_{\|}} \mathrm{C}\left\{\overline{\mathrm{H}}_{\mathrm{p}}-\frac{\mathrm{v}_{\|} \mathrm{f}_{0}}{\Omega \mathrm{T}}\left(\frac{\mathrm{M}_{\mathrm{i}} \mathrm{v}^{2}}{2 \mathrm{~T}}-\frac{5}{2}\right)\left\langle\frac{\overrightarrow{\mathrm{B}} \cdot \nabla \psi \times \nabla \mathrm{B}}{\overrightarrow{\mathrm{B}} \cdot \nabla \mathrm{B}}\right\rangle \frac{\partial \mathrm{T}}{\partial \psi}\right\}\right\rangle,
$$

where conservation of momentum for ion-ion collisions, $\mathrm{C}\left\{\mathrm{v}_{\|} \mathrm{f}_{0}\right\}=0$, is employed to obtain the final form. As the flux surface average sums over both well branches, omnigeneity is used to neglect $\Sigma \partial \mathrm{F}_{0} / \partial \alpha \simeq 0$ and $\Sigma \partial \tilde{\mathrm{J}} / \partial \alpha \simeq 0$ as small compared to the large term retained. Within a geometric factor the preceding constraint equation is the same found as for a tokamak and for an omnigenous flux surface (Landreman \& Catto, 2012).

To form the parallel flow and parallel current density the trapped equation must also be solved. In this case, the kinetic equation must be written in a form with the $\Delta$ terms appearing in the large parallel streaming term. Recalling (5.9) means that a non-omnigeous flux surface

$$
\overrightarrow{\overrightarrow{\mathrm{v}}_{\mathrm{d}} \cdot \nabla \psi}=\overline{\mathrm{v}_{\|} \overrightarrow{\mathrm{b}} \cdot \nabla \Delta_{\mathrm{t}}} \neq 0 \text {, }
$$

so $\overline{v_{\|} \vec{b} \cdot \nabla \Delta_{t}}$ must be retained. Therefore, using

gives

$$
\mathrm{H}_{\mathrm{t}}=\mathrm{h}_{\mathrm{t}}+\Delta_{\mathrm{t}} \partial \mathrm{f}_{0} / \partial \psi
$$

$$
\overline{\mathrm{H}}_{\mathrm{t}}=\overline{\mathrm{h}}_{\mathrm{t}}+\bar{\Delta}_{\mathrm{t}} \partial \mathrm{f}_{0} / \partial \psi
$$

(and not $\left.\overline{\mathrm{H}}_{\mathrm{t}}=\overline{\mathrm{h}}_{\mathrm{t}}\right)$, where both $\overline{\mathrm{H}}_{\mathrm{t}}=\overline{\mathrm{H}}_{\mathrm{t}}(\psi, \alpha, \mathrm{v}, \lambda)$ and $\overline{\mathrm{h}}_{\mathrm{t}}=\overline{\mathrm{h}}_{\mathrm{t}}(\psi, \alpha, \mathrm{v}, \lambda)$ are even functions of $\mathrm{v}_{\|}$, with $\overrightarrow{\mathrm{b}} \cdot \nabla \overline{\mathrm{H}}_{\mathrm{t}}=0=\overrightarrow{\mathrm{b}} \cdot \nabla \overline{\mathrm{h}}_{\mathrm{t}}, \mathrm{h}_{\mathrm{t}}-\overline{\mathrm{h}}_{\mathrm{t}}=\tilde{\mathrm{h}}_{\mathrm{t}}$, and $\mathrm{H}_{\mathrm{t}}-\overline{\mathrm{H}}_{\mathrm{t}}=\tilde{\mathrm{H}}_{\mathrm{t}}$. Moreover, 
where

$$
\tilde{\mathrm{H}}_{\mathrm{t}}=\tilde{\mathrm{h}}_{\mathrm{t}}+\left(\Delta_{\mathrm{t}}-\bar{\Delta}_{\mathrm{t}}\right) \partial \mathrm{f}_{0} / \partial \psi
$$

$$
\tilde{\mathrm{h}}_{\mathrm{t}} \sim \Delta_{\mathrm{t}} \partial \mathrm{f}_{0} / \partial \psi \lesssim \sqrt{\delta} \rho_{\mathrm{pi}} \mathrm{f}_{0} / \mathrm{a}
$$

The trapped equation,

$$
\mathrm{C}\left\{\mathrm{h}_{\mathrm{t}}\right\}-\overrightarrow{\mathrm{v}}_{\mathrm{d}} \cdot \nabla \mathrm{h}_{\mathrm{t}}=\mathrm{v}_{\|} \overrightarrow{\mathrm{b}} \cdot \nabla \tilde{\mathrm{H}}_{\mathrm{t}}=\mathrm{v}_{\|} \overrightarrow{\mathrm{b}} \cdot \nabla\left(\mathrm{h}_{\mathrm{t}}+\Delta_{\mathrm{t}} \partial \mathrm{f}_{0} / \partial \psi\right)
$$

is transit averaged and $\vec{v}_{d} \cdot \nabla h_{t} \rightarrow-\omega \partial h_{t} / \partial \alpha$ employed to obtain the combined $\sqrt{v}$ and $1 / v$ form

$$
\omega \partial \overline{\mathrm{h}}_{\mathrm{t}} / \partial \alpha+\overline{\mathrm{C}\left\{\overline{\mathrm{h}}_{\mathrm{t}}\right\}}=\overline{\mathrm{v}_{\|} \overrightarrow{\mathrm{b}} \cdot \nabla \Delta_{\mathrm{t}}} \partial \mathrm{f}_{0} / \partial \psi,
$$

where in the small tangential drift and collision terms on the left the lowest order omnigenous trapped orbits are used in the averages so $\tilde{h}_{t}$ does not contribute. In the superbanana plateau regime the magnetic drift must be retained, but as this only alters the even part of the perturbed distribution function it does not affect the parallel flow and bootstrap current.

The assumption that streaming dominates means $(\omega+v)<\mathrm{v}_{\|} / \mathrm{qR} \sim \mathrm{v}_{\|} / \mathrm{R}$. As a result,

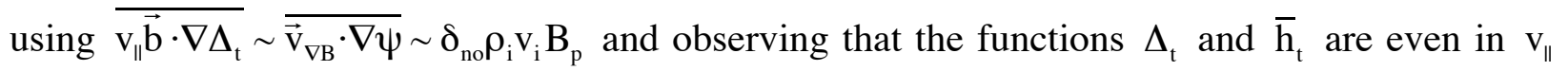
yields the estimate

$$
\frac{\overline{\mathrm{h}}_{\mathrm{t}}}{\mathrm{f}_{0}} \lesssim \frac{\delta_{\mathrm{no}} \mathrm{v}_{\mathrm{i}} \rho_{\mathrm{i}}}{\left(\omega+v_{\mathrm{i}}\right) \mathrm{Ra}}<<1,
$$

where $\mathrm{v}_{\|}^{2} \sim \delta \mathrm{v}_{\mathrm{i}}^{2}$ is not used as the non-omnigenous $\nabla \mathrm{B}$ drift dominates in (5.6) with $\Delta \lesssim \mathrm{V}_{\|} \mathrm{RB} / \Omega$ from (3.13). The unaveraged form of the trapped equation (6.18) suggests

$$
\frac{\tilde{\mathrm{H}}_{\mathrm{t}}}{\tilde{\mathrm{h}}_{\mathrm{t}}} \sim \frac{\left(\omega+v_{\mathrm{i}}\right) \mathrm{R}}{\mathrm{v}_{\mathrm{i}} \sqrt{\delta}} \ll<,
$$

so that $\tilde{\mathrm{H}}_{\mathrm{t}}$ can be neglected as small in (6.16), giving

$$
\tilde{\mathrm{h}}_{\mathrm{t}}=-\left(\Delta_{\mathrm{t}}-\bar{\Delta}_{\mathrm{t}}\right) \partial \mathrm{f}_{0} / \partial \psi \simeq-\Delta_{\mathrm{t}} \partial \mathrm{f}_{0} / \partial \psi
$$

as $\bar{\Delta}_{\mathrm{t}}$ is small. Allowing $\overline{\mathrm{h}}_{\mathrm{t}} \sim \tilde{\mathrm{h}}_{\mathrm{t}},(6.17)$ and (6.20) yield another constraint on $\delta_{\mathrm{no}} \ll \delta$ of

$$
\delta_{\mathrm{no}} \sqrt{\delta} \sim \frac{\left(\omega+v_{\mathrm{i}}\right) \mathrm{R}}{\mathrm{v}_{\mathrm{i}}} \sim \frac{\rho_{\mathrm{pi}}}{\mathrm{a}}+\frac{\mathrm{R}}{\lambda} \ll 1,
$$

where the mean free path is $\lambda \sim v_{i} / \nu_{i}>>R$ and $v_{i}>>R$.

Just as the departure from an omnigenous flux surface makes $\overline{v_{\|} \vec{b} \cdot \nabla \Delta_{t}} \neq 0$, the transit averaged radial trapped step $\bar{\Delta}_{\mathrm{t}}$ no longer vanishes as

$$
\bar{\Delta}_{\mathrm{t}}=\frac{\overline{\mathrm{v}_{\|} \overrightarrow{\mathrm{B}} \cdot \nabla \psi \times \nabla \mathrm{B}}}{\Omega \overrightarrow{\mathrm{B}} \cdot \nabla \mathrm{B}}-\frac{\overline{\partial \tilde{\mathrm{J}}}}{\partial \alpha}=\frac{\overline{\mathrm{v}_{\|} \partial \mathrm{F}_{0}}}{\Omega \partial \alpha}-\frac{\overline{\partial \tilde{\mathrm{J}}}}{\partial \alpha} \neq 0 .
$$

The trapped $\mathrm{F}_{0}$ and $\tilde{\mathbf{J}}$ now depend on the field line label $\alpha$ so that $\left\langle\mathrm{Bv}_{\|}^{-1} \partial \mathrm{F}_{0} / \partial \alpha\right\rangle \neq 0$ and $\langle\partial \tilde{\mathbf{J}} / \partial \alpha\rangle \neq 0$. Moreover, the omnigenous choice for the lower limit $\overline{\mathrm{B}}$ of $\tilde{\mathbf{J}}$ for the trapped is no longer quite right when the maximum field on a field line varies from one end to the other and also depends on the field line.

As $\bar{h}_{t}$ is even in $v_{\|}$it will not contribute to the parallel ion flow or the parallel current density, and there is no need to evaluate it to determine the bootstrap current. 
To find the contributions to the parallel ion flow and parallel current density the passing constraint (6.12) must be solved. The solution procedure uses the linearized model like particle collision operator of Kovrizhnikh \& Connor (Rosenbluth et al. 1972; Connor et al. 1973),

$$
\mathrm{C}\{\mathrm{g}\}=v \mathrm{Q}(\mathrm{v})\left[\mathrm{L}\{\mathrm{g}\}+\frac{\mathrm{M}_{\mathrm{i}}}{\mathrm{T}} \mathrm{uv}_{\|} \mathrm{f}_{0}\right]=v \mathrm{Q}(\mathrm{v}) \mathrm{L}\left\{\mathrm{g}-\frac{\mathrm{M}_{\mathrm{i}}}{\mathrm{T}} \mathrm{uv}_{\|} \mathrm{f}_{0}\right\},
$$

where $\mathrm{L}$ is the Lorentz operator

$$
\mathrm{L}\{\mathrm{g}\}=\frac{1}{2} \nabla_{\mathrm{v}} \cdot\left[\left(\mathrm{v}^{2} \overrightarrow{\mathrm{I}}-\overrightarrow{\mathrm{v}} \overrightarrow{\mathrm{v}}\right) \cdot \nabla_{\mathrm{v}} \mathrm{g}\right]=\frac{2 \mathrm{~B}_{0}}{\mathrm{Bv}^{2}} \mathrm{v}_{\|} \frac{\partial}{\partial \lambda}\left(\lambda \mathrm{v}_{\|} \frac{\partial \mathrm{g}}{\partial \lambda}\right),
$$

with $\mathrm{L}\left\{\mathrm{v}_{\|} \mathrm{f}_{0}\right\}=-\mathrm{v}_{\|} \mathrm{f}_{0}$, and $\mathrm{u}$ the term retained to preserve collisional momentum conservation,

$$
\mathrm{u}=3 \mathrm{~T} \int \mathrm{d}^{3} \mathrm{vQv} \mathrm{v}_{\|} \mathrm{g} / \mathrm{M}_{\mathrm{i}} \int \mathrm{d}^{3} \mathrm{vQv} \mathrm{v}^{2} \mathrm{f}_{0} \text {. }
$$

The function $\mathrm{Q}$ is defined as

$$
\mathrm{Q}(\mathrm{x})=\frac{1}{\mathrm{x}^{3}}\left[\left(1-\frac{1}{2 \mathrm{x}^{2}}\right) \operatorname{erf}(\mathrm{x})+\frac{1}{2 \mathrm{x}} \frac{\mathrm{d}}{\mathrm{dx}} \operatorname{erf}(\mathrm{x})\right],
$$

with $\mathrm{x}=\mathrm{v}\left(\mathrm{M}_{\mathrm{i}} / 2 \mathrm{~T}\right)^{1 / 2}$, and $\operatorname{erf}(\mathrm{x})=2 \pi^{-1 / 2} \int_{0}^{\mathrm{x}} \mathrm{dte} \mathrm{t}^{-\mathrm{t}^{2}}$ the error function. For ions

$$
v_{\mathrm{i}} \equiv \frac{\sqrt{2} \pi \mathrm{Z}^{4} \mathrm{e}^{4} \mathrm{n}_{\mathrm{i}} \ell \mathrm{n} \Lambda}{\mathrm{M}_{\mathrm{i}}^{1 / 2} \mathrm{~T}_{\mathrm{i}}^{3 / 2}} \rightarrow \frac{3 \sqrt{2 \pi}}{4} v_{\mathrm{ii}}
$$

with $v_{i i}=4 \sqrt{\pi} Z^{4} e^{4} n_{i} \ell n \Lambda / 3 M_{i}^{1 / 2} T_{i}^{3 / 2}$.

The flux surface averaged passing form is then

$$
\left\langle\frac{\mathrm{B}}{\mathrm{v}_{\|}} \mathrm{C}\{\mathrm{g}\}\right\rangle=\frac{2 v \mathrm{QB}_{0}}{\mathrm{v}^{2}} \frac{\partial}{\partial \lambda}\left[\lambda\left\langle\mathrm{v}_{\|} \frac{\partial}{\partial \lambda}\left(\mathrm{g}-\frac{\mathrm{M}_{\mathrm{j}}}{\mathrm{T}} \mathrm{uv}_{\|} \mathrm{f}_{0}\right)\right\rangle\right],
$$

where based on constraint (6.12)

$$
\mathrm{g}=\overline{\mathrm{H}}_{\mathrm{p}}-\frac{\mathrm{v}_{\|} \mathrm{f}_{0}}{\Omega \mathrm{T}}\left(\frac{\mathrm{M}_{\mathrm{i}} \mathrm{v}^{2}}{2 \mathrm{~T}}-\frac{5}{2}\right)\left\langle\frac{\overrightarrow{\mathrm{B}} \cdot \nabla \psi \times \nabla \mathrm{B}}{\Omega \overrightarrow{\mathrm{B}} \cdot \nabla \mathrm{B}}\right\rangle \frac{\partial \mathrm{T}}{\partial \psi} .
$$

Integrating from $\lambda=0$, and using $\mathrm{v}_{\|} \partial \mathrm{v}_{\|} / \partial \lambda=-\mathrm{Bv}^{2} / 2 \mathrm{~B}_{0}$ leads to

$$
\frac{\left\langle\mathrm{v}_{\|}\right\rangle_{\mathrm{p}}}{\mathrm{f}_{0}} \frac{\partial \overline{\mathrm{H}}_{\mathrm{p}}}{\partial \lambda}=-\frac{\mathrm{M}_{\mathrm{i}} \mathrm{v}^{2}}{2 \mathrm{~TB}_{0}}\langle\mathrm{Bu}\rangle-\frac{\mathrm{v}^{2}}{2 \Omega_{0} \mathrm{~T}}\left(\frac{\mathrm{M}_{\mathrm{i}} \mathrm{v}^{2}}{2 \mathrm{~T}}-\frac{5}{2}\right)\left\langle\frac{\overrightarrow{\mathrm{B}} \cdot \nabla \psi \times \nabla \mathrm{B}}{\overrightarrow{\mathrm{B}} \cdot \nabla \mathrm{B}}\right\rangle \frac{\partial \mathrm{T}}{\partial \psi},
$$

where $\Omega_{0}=\mathrm{ZeB}_{0} / \mathrm{M}_{\mathrm{i}} \mathrm{c}$ and

$$
\langle\mathrm{Bu}\rangle=3 \mathrm{TM}_{\mathrm{i}}^{-1} \mathrm{~B}^{-1} \int \mathrm{d}^{3} \mathrm{vQv} \mathrm{v}_{\|}\left\langle\mathrm{B}^{2} \mathrm{~g}\right\rangle / \int \mathrm{d}^{3} \mathrm{vQv} \mathrm{v}^{2} \mathrm{f}_{0} .
$$

The second $\lambda$ integration is performed to make $g$ vanish at the trapped-passing boundary $\lambda=\mathrm{B}_{0} / \widehat{\mathrm{B}}$, with $\widehat{\mathrm{B}}$ the maximum value of $\mathrm{B}$ on a flux surface, to find

$$
\overline{\mathrm{H}}_{\mathrm{pi}}=\left\langle\frac{\overrightarrow{\mathrm{B}} \cdot \nabla \psi \times \nabla \mathrm{B}}{\overrightarrow{\mathrm{B}} \cdot \nabla \mathrm{B}}\right\rangle\left(\frac{\mathrm{M}_{\mathrm{i}} \mathrm{v}^{2}}{2 \mathrm{~T}_{\mathrm{i}}}-1.33\right) \frac{\sigma \mathrm{vf}_{0 \mathrm{i}} \partial \mathrm{T}_{\mathrm{i}}^{\mathrm{B}_{0} / \widehat{\mathrm{B}}}}{2 \Omega_{0} \mathrm{~T}_{\mathrm{i}} \partial \psi} \int_{\lambda} \frac{\mathrm{d} \lambda^{\prime}}{\left\langle\sqrt{1-\lambda^{\prime} \mathrm{B} / \mathrm{B}_{0}}\right\rangle} .
$$

Using (6.9), (6.14), (6.22), and (6.34) gives

$$
\frac{\mathrm{f}_{1 \mathrm{i}}}{\mathrm{f}_{0 \mathrm{i}}}-\frac{\mathrm{Ze} \phi}{\mathrm{T}_{\mathrm{i}}}=\frac{\mathrm{h}_{\mathrm{i}}}{\mathrm{f}_{0 \mathrm{i}}}=\frac{\overline{\mathrm{H}}_{\mathrm{pi}}+\overline{\mathrm{h}}_{\mathrm{ti}}+\tilde{\mathrm{H}}_{\mathrm{ti}}+\tilde{\mathrm{H}}_{\mathrm{pi}}}{\mathrm{f}_{0 \mathrm{i}}}-\left[\frac{\Delta_{\mathrm{p}}}{\mathrm{f}_{0 \mathrm{i}}}+\frac{\left(\Delta_{\mathrm{t}}-\bar{\Delta}_{\mathrm{t}}\right)}{\mathrm{f}_{0 \mathrm{i}}}\right] \frac{\partial \mathrm{f}_{0 \mathrm{i}}}{\partial \psi} \simeq \frac{\overline{\mathrm{H}}_{\mathrm{pi}}+\overline{\mathrm{h}}_{\mathrm{ti}}+\tilde{\mathrm{H}}_{\mathrm{pi}}}{\mathrm{f}_{0 \mathrm{i}}}-\frac{\Delta}{\mathrm{f}_{0 \mathrm{i}}} \frac{\partial \mathrm{f}_{0 \mathrm{i}}}{\partial \psi},
$$

where the last form neglects $\tilde{\mathrm{H}}_{\mathrm{ti}}$ and $\bar{\Delta}_{\mathrm{t}}$, and uses $\Delta=\Delta_{\mathrm{p}}+\Delta_{\mathrm{t}}$. Inserting $\Delta$ and the solution for $\overline{\mathrm{H}}_{\mathrm{pi}}$ obtained by Landreman and Catto (2012) with the geometric and notational replacements 


$$
\frac{\mathrm{MI}+\mathrm{NG}}{\mathrm{M}-\mathrm{qN}} \rightarrow\left\langle\frac{\overrightarrow{\mathrm{B}} \cdot \nabla \psi \times \nabla \mathrm{B}}{\overrightarrow{\mathrm{B}} \cdot \nabla \mathrm{B}}\right\rangle
$$

yields

$$
\begin{aligned}
& \frac{\mathrm{f}_{1 \mathrm{i}}}{\mathrm{f}_{0 \mathrm{i}}}=\frac{\overline{\mathrm{H}}_{\mathrm{ti}}+\tilde{\mathrm{H}}_{\mathrm{pi}}}{\mathrm{f}_{0 \mathrm{i}}}+\frac{\mathrm{Ze} \phi}{\mathrm{T}_{\mathrm{i}}}-\left(\frac{\mathrm{v}_{\| l} \overrightarrow{\mathrm{B}} \cdot \nabla \psi \times \nabla \mathrm{B}}{\Omega \overrightarrow{\mathrm{B}} \cdot \nabla \mathrm{B}}-\frac{\partial \tilde{\mathrm{J}}}{\partial \alpha}\right)\left[\frac{1 \partial \mathrm{p}_{\mathrm{i}}}{\mathrm{p}_{\mathrm{i}} \partial \psi}+\frac{\mathrm{Ze} \partial \Phi}{\mathrm{T}_{\mathrm{i}} \partial \psi}+\left(\frac{\mathrm{M}_{\mathrm{i}} \mathrm{v}^{2}}{2 \mathrm{~T}_{\mathrm{i}}}-\frac{5}{2}\right) \frac{1 \partial \mathrm{T}_{\mathrm{i}}}{\mathrm{T}_{\mathrm{i}} \partial \psi}\right] \\
& +\left\langle\frac{\overrightarrow{\mathrm{B}} \cdot \nabla \psi \times \nabla \mathrm{B}}{\overrightarrow{\mathrm{B}} \cdot \nabla \mathrm{B}}\right\rangle\left(\frac{\mathrm{M}_{\mathrm{i}} \mathrm{v}^{2}}{2 \mathrm{~T}_{\mathrm{i}}}-1.33\right) \frac{\sigma v \mathrm{H}\left(\mathrm{B}_{0} / \widehat{\mathrm{B}}-\lambda\right) \partial \mathrm{T}_{\mathrm{i}}}{2 \Omega_{0} \mathrm{~T}_{\mathrm{i}}} \partial \psi \int_{\lambda}^{\mathrm{B}_{0} / \widehat{\mathrm{B}}} \frac{\mathrm{d} \lambda^{\prime}}{\left\langle\sqrt{1-\lambda^{\prime} \mathrm{B} / \mathrm{B}_{0}}\right\rangle}
\end{aligned}
$$

where $\overline{\mathrm{H}}_{\mathrm{ti}}$ and $\tilde{\mathrm{H}}_{\mathrm{pi}}$ are unimportant for parallel flow as they must be even functions of $\mathrm{v}_{\|}$with $\tilde{\mathrm{H}}_{\mathrm{pi}}<<\overline{\mathrm{H}}_{\mathrm{pi}}$ with $\overline{\mathrm{H}}_{\mathrm{pi}}$ odd in $\mathrm{v}_{\|}$. A Heaviside step function $\mathrm{H}\left(\mathrm{B}_{0} / \widehat{\mathrm{B}}-\lambda\right)$, with value one for $\lambda<\mathrm{B}_{0} / \widehat{\mathrm{B}}$ and vanishing otherwise, is inserted to combine the trapped and passing forms.

The next section evaluates the parallel ion flow velocity and parallel current density associated with the perturbed distribution function $\mathrm{f}_{1 \mathrm{i}}$ given by (6.37).

\section{Flow and current density for a weak departure from omnigeneity}

Determining the parallel ion velocity requires evaluating $\int \mathrm{d}^{3} \mathrm{vv}_{\|} \mathrm{h}_{\mathrm{i}}=\int \mathrm{d}^{3} \mathrm{vv}_{\|}\left(\mathrm{h}_{\mathrm{pi}}+\mathrm{h}_{\mathrm{ti}}\right)$. The procedure is straightforward to summarize. First, as $\overline{\mathrm{H}}_{\mathrm{ti}}$ and $\tilde{\mathrm{H}}_{\mathrm{pi}}$ are even in $\mathrm{v}_{\|}$,

$$
\int \mathrm{d}^{3} \mathrm{vv}_{\|}\left(\overline{\mathrm{H}}_{\mathrm{ti}}+\tilde{\mathrm{H}}_{\mathrm{pi}}\right)=0 \text {. }
$$

Also, integrating

$$
M_{i} \int d^{3} v v_{\|}^{2} \frac{\partial f_{0}}{\partial \psi}=\left(\frac{\partial p}{\partial \psi}+\operatorname{Zen} \frac{\partial \Phi}{\partial \psi}\right) .
$$

In addition, recalling the omnigenous result (5.12), using $\mathrm{d}^{3} \mathrm{v} \rightarrow 2 \pi\left(\mathrm{Bv}^{2} / \mathrm{B}_{0} \xi\right) \mathrm{dvd} \lambda$ as all integrals are even in $\mathrm{v}_{\| \mid}$, and following the procedure below (49) in Landreman \& Catto (2012),

$$
\begin{aligned}
& \mathrm{M}_{\mathrm{i}} \int \mathrm{d}^{3} \mathrm{vv}_{\|} \frac{\partial \tilde{\mathrm{J}} \partial \mathrm{f}_{0}}{\partial \alpha \partial \psi}=\mathrm{M}_{\mathrm{i}} \int \mathrm{d}^{3} \mathrm{v} \mathrm{v}_{\|} \frac{\partial \mathrm{f}_{0}}{\partial \psi}\left[\frac{\mathrm{v}_{\|}}{\Omega\left(\partial \mathrm{F}_{0}\right.}+\mathrm{v}^{2} \int_{\overline{\mathrm{B}}}^{\mathrm{B}} \mathrm{dB}^{\prime} \frac{\partial \mathrm{F}_{0}^{\prime}\left(2-\lambda \mathrm{B}^{\prime} / \mathrm{B}_{0}\right)}{\partial \alpha 2 \Omega^{\prime} \mathrm{B}^{\prime} \mathrm{v}_{\|}^{\prime}}\right]=\frac{1 \partial \mathrm{F}_{0}}{\Omega \partial \alpha}\left(\frac{\partial \mathrm{p}}{\partial \psi}+\operatorname{Zen} \frac{\partial \Phi}{\partial \psi}\right) \\
& -\frac{\pi \mathrm{M}_{\mathrm{i}} \mathrm{B}}{\Omega_{0}} \int_{0}^{\infty} \mathrm{dvv}^{4} \frac{\partial \mathrm{f}_{0}}{\partial \psi} \int_{\mathrm{B}}^{\mathrm{B}} \frac{\mathrm{dB}^{\prime} \partial \mathrm{F}_{0}^{\prime}}{\mathrm{B}^{\prime 2} \partial \alpha} \int_{0}^{\mathrm{B}_{0} / \mathrm{B}^{\prime}} \mathrm{d} \lambda \frac{\left(2-\lambda \mathrm{B}^{\prime} / \mathrm{B}_{0}\right)}{\sqrt{1-\lambda \mathrm{B}^{\prime} / \mathrm{B}_{0}}}=\left(\frac{1 \partial \mathrm{F}_{0}}{\Omega \alpha}-\frac{\mathrm{W}}{\Omega}\right)\left(\frac{\partial \mathrm{p}}{\partial \psi}+\operatorname{Zen} \frac{\partial \Phi}{\partial \psi}\right)
\end{aligned},
$$

where near omnigeneity is used to write $\mathrm{W}$ as

$$
\mathrm{W} \equiv 2 \mathrm{~B}^{2} \int_{\mathrm{B}}^{\widehat{\mathrm{B}}} \frac{\mathrm{dB}^{\prime}}{\mathrm{B}^{\prime 3}} \frac{\partial \mathrm{F}_{0}^{\prime}}{\partial \alpha} .
$$

with the upper limit of $\widehat{B}=B_{\max }$ to integrate over all $\mathrm{B}^{\prime}$. Moreover, (6.34) yields

$$
\mathrm{M}_{\mathrm{i}} \int \mathrm{d}^{3} \mathrm{vV}_{\|} \overline{\mathrm{H}}_{\mathrm{pi}}=\left\langle\frac{\overrightarrow{\mathrm{B}} \cdot \nabla \psi \times \nabla \mathrm{B}}{\overrightarrow{\mathrm{B}} \cdot \nabla \mathrm{B}}\right\rangle \frac{\mathrm{Bf}_{\mathrm{c}} \mathrm{n}_{\mathrm{i}} \partial \mathrm{T}_{\mathrm{i}}}{\mathrm{B}_{0} \Omega_{0} \partial \psi},
$$

where $f_{c}$ is the usual effective passing fraction defined by

$$
\mathrm{f}_{\mathrm{c}}=\frac{3}{4} \int_{0}^{\mathrm{B}_{0} / \widehat{\mathrm{B}}} \frac{\mathrm{d} \lambda \lambda}{\left\langle\sqrt{1-\lambda \mathrm{B} / \mathrm{B}_{0}}\right\rangle} \rightarrow 1-1.46 \sqrt{\delta}
$$

The preceding evaluation makes use of 


$$
\int_{0}^{\mathrm{B}_{0} / \hat{\mathrm{B}}} \mathrm{d} \lambda \int_{\lambda}^{\mathrm{B}_{0} / \hat{\mathrm{B}}} \frac{\mathrm{d} \lambda^{\prime}}{\left\langle\sqrt{1-\lambda^{\prime} \mathrm{B} / \mathrm{B}_{0}}\right\rangle}=\int_{0}^{\mathrm{B}_{0} / \hat{\mathrm{B}}} \frac{\mathrm{d} \lambda^{\prime}}{\left\langle\sqrt{1-\lambda^{\prime} \mathrm{B} / \mathrm{B}_{0}}\right\rangle} \int_{0}^{\lambda^{\prime}} \mathrm{d} \lambda=\int_{0}^{\mathrm{B}_{0} / \hat{\mathrm{B}}} \frac{\mathrm{d} \lambda^{\prime} \lambda^{\prime}}{\left\langle\sqrt{1-\lambda^{\prime} \mathrm{B} / \mathrm{B}_{0}}\right\rangle}=\frac{4}{3} \mathrm{f}_{\mathrm{c}},
$$

and

$$
\int \mathrm{d}^{3} \mathrm{vv}_{\|} \sigma v f_{0 \mathrm{i}}\left(\frac{\mathrm{M}_{\mathrm{i}} \mathrm{v}^{2}}{2 \mathrm{~T}_{\mathrm{i}}}-1.33\right)=\frac{2 \pi \mathrm{B}}{\mathrm{B}_{0}} \int_{0}^{\mathrm{B}_{0} / \widehat{\mathrm{B}}} \mathrm{d} \lambda \int_{0}^{\infty} \mathrm{dvf}_{0 \mathrm{i}} \mathrm{v}^{4}\left(\frac{\mathrm{M}_{\mathrm{i}} \mathrm{v}^{2}}{2 \mathrm{~T}_{\mathrm{i}}}-1.33\right)=1.17 \frac{3 \mathrm{p}_{\mathrm{i}} \mathrm{B}}{2 \mathrm{M}_{\mathrm{i}} \mathrm{B}_{0}}
$$

Combining these results, the parallel ion velocity, $\mathrm{V}_{\text {li }}$, for a non-optimized stellarator is

$$
\mathrm{n}_{\mathrm{i}} \mathrm{V}_{\| \mathrm{i}}=\int \mathrm{d}^{3} \mathrm{vv}_{\| l} \mathrm{f}_{1 \mathrm{i}}=\frac{-\mathrm{c}}{\mathrm{ZeB}}\left[\left(\left\langle\frac{\overrightarrow{\mathrm{B}} \cdot \nabla \psi \times \nabla \mathrm{B}}{\overrightarrow{\mathrm{B}} \cdot \nabla \mathrm{B}}\right\rangle+\mathrm{W}\right)\left(\frac{\partial \mathrm{p}_{\mathrm{i}}}{\partial \psi}+\operatorname{Zen}_{\mathrm{i}} \frac{\partial \Phi}{\partial \psi}\right)-1.17\left\langle\frac{\overrightarrow{\mathrm{B}} \cdot \nabla \psi \times \nabla \mathrm{B}}{\overrightarrow{\mathrm{B}} \cdot \nabla \mathrm{B}}\right\rangle \frac{\mathrm{B}^{2} \mathrm{f}_{\mathrm{c}} \mathrm{n}_{\mathrm{i}} \partial \mathrm{T}_{\mathrm{i}}}{\left\langle\mathrm{B}^{2}\right\rangle \partial \psi}\right],
$$

where $\left\langle\partial \mathrm{F}_{0} / \partial \alpha\right\rangle$ is neglected as the departure from omnigeneity is weak. This result agrees Helander et al. (2017), but expresses the geometrical factors in a more compact way thanks to the assumption of near-omnigeniety. It thus also reduces to the omnigenous limit found by Landreman and Catto (2012) (within minor notational differences).

Checking $\nabla \cdot \vec{V}=0$ by recalling (4.6), and using

$$
\overrightarrow{\mathrm{B}} \cdot \nabla\left(\int \mathrm{d}^{3} \mathrm{v} \frac{\mathrm{v}_{\|} \partial \tilde{\mathrm{J}} \partial \mathrm{f}_{0}}{\mathrm{~B} \partial \alpha \partial \psi}\right)=\overrightarrow{\mathrm{B}} \cdot \nabla \mathrm{B} \frac{\partial}{\partial \alpha} \int \mathrm{d}^{3} \mathrm{v} \frac{\mathrm{v}_{\|} \partial \mathrm{f}_{0}}{\mathrm{~B} \partial \psi} \frac{\partial \tilde{\mathrm{J}}}{\partial \mathrm{B}}=\frac{\mathrm{M}_{\mathrm{i}} \mathrm{c}}{\mathrm{Ze}} \overrightarrow{\mathrm{b}} \cdot \nabla \mathrm{B} \frac{\partial}{\partial \alpha}\left(\frac{1}{\overrightarrow{\mathrm{b}} \cdot \nabla \mathrm{B}}\right) \int \mathrm{d}^{3} \mathrm{vv}_{\|}^{2} \frac{\partial \mathrm{f}_{0}}{\partial \psi},
$$

gives the required result

$$
\mathrm{n}_{\mathrm{i}} \overrightarrow{\mathrm{B}} \cdot \nabla\left(\mathrm{V}_{\| \mathrm{i}} / \mathrm{B}\right)=-\frac{\mathrm{c}}{\mathrm{Ze}}\left(\frac{\partial \mathrm{p}_{\mathrm{i}}}{\partial \psi}+\operatorname{Zen}_{\mathrm{i}} \frac{\partial \Phi}{\partial \psi}\right) \overrightarrow{\mathrm{B}} \cdot \nabla(\mathrm{U} / \mathrm{B})=-\frac{\mathrm{c}}{\mathrm{Ze}}\left(\frac{\partial \mathrm{p}_{\mathrm{i}}}{\partial \psi}+\operatorname{Zen}_{\mathrm{i}} \frac{\partial \Phi}{\partial \psi}\right) \nabla \cdot\left(\mathrm{B}^{-2} \overrightarrow{\mathrm{B}} \times \nabla \psi\right) .
$$

Writing

$$
\mathrm{V}_{\text {lli }}=\mathrm{V}_{\text {lli }}^{\mathrm{PS}}+\mathrm{B}\left\langle\mathrm{B} \mathrm{V}_{\mathrm{li}}\right\rangle /\left\langle\mathrm{B}^{2}\right\rangle,
$$

and using lowest order omnigeneity gives

$$
\mathrm{n}_{\mathrm{i}}\left\langle\mathrm{B} V_{\text {li }}\right\rangle=-\frac{\mathrm{c}}{\mathrm{Ze}}\left\langle\frac{\overrightarrow{\mathrm{B}} \cdot \nabla \psi \times \nabla \mathrm{B}}{\overrightarrow{\mathrm{B}} \cdot \nabla \mathrm{B}}\right\rangle\left(\frac{\partial \mathrm{p}_{\mathrm{i}}}{\partial \psi}+\operatorname{Zen}_{\mathrm{i}} \frac{\partial \Phi}{\partial \psi}-1.17 \mathrm{f}_{\mathrm{c}} \mathrm{n}_{\mathrm{i}} \frac{\partial \mathrm{T}_{\mathrm{i}}}{\partial \psi}\right),
$$

so that the Pfirsch-Schlüter flow is

$$
\mathrm{n}_{\mathrm{i}} \mathrm{V}_{\text {li }}^{\mathrm{PS}}=-\frac{\mathrm{c}}{\mathrm{ZeB}}\left(\frac{\partial \mathrm{p}_{\mathrm{i}}}{\partial \psi}+\mathrm{Zen}_{\mathrm{i}} \frac{\partial \Phi}{\partial \psi}\right)\left[\left(1-\frac{\mathrm{B}^{2}}{\left\langle\mathrm{~B}^{2}\right\rangle}\right)\left\langle\frac{\overrightarrow{\mathrm{B}} \cdot \nabla \psi \times \nabla \mathrm{B}}{\overrightarrow{\mathrm{B}} \cdot \nabla \mathrm{B}}\right\rangle+\mathrm{W}\right] .
$$

Nearly all the complicated geometric behavior is in the Pfirsch-Schlüter flow.

The treatment for the electrons is similar, but it must keep unlike electron-ion collisions as well as like collisions. The procedure is the same as for a tokamak, as summarized in Helander \& Sigmar (2002), and an omnigenous stellarator, as employed in Landreman \& Catto (2012). The results are consistent with the more geometrically involved general stellarator results of Helander et al. (2011 \& 2017).

The bootstrap current for weakly non-optimized stellarators is most easily found from the Landreman \& Catto (2012) form by recalling the replacement (3.13). Within minor notational differences the result is

$$
\left\langle\mathrm{BJ}_{\|}\right\rangle=\frac{\mathrm{c}\left(\mathrm{Z}^{2}+2.2 \mathrm{Z}+0.75\right)\left(1-\mathrm{f}_{\mathrm{c}}\right)}{\mathrm{Z}(\mathrm{Z}+\sqrt{2})}\left\langle\frac{\overrightarrow{\mathrm{B}} \cdot \nabla \psi \times \nabla \mathrm{B}}{\overrightarrow{\mathrm{B}} \cdot \nabla \mathrm{B}}\right\rangle\left[\frac{\partial \mathrm{p}_{\mathrm{i}}}{\partial \psi}+\frac{\partial \mathrm{p}_{\mathrm{e}}}{\partial \psi}-\frac{(2.1 \mathrm{Z}+0.88) \mathrm{n}_{\mathrm{e}} \partial \mathrm{T}_{\mathrm{e}}}{\left(\mathrm{Z}^{2}+2.2 \mathrm{Z}+0.75\right) \partial \psi}-\frac{1.17 \mathrm{n}_{\mathrm{e}} \partial \mathrm{T}_{\mathrm{i}}}{\mathrm{Z} \quad \partial \psi}\right] .
$$


Only the geometrical coefficients of the bootstrap current differ from the forms of Landreman \& Catto (2012) and Helander et al. (2017). When normalized to the axisymmetric tokamak result $(\mathrm{N}=0$ in the quasisymetric limit) a geometric ratio is obtained,

$$
\frac{\left\langle\mathrm{BJ}_{\|}\right\rangle}{\left\langle\mathrm{BJ}_{\|}\right\rangle_{\text {tok }}}=\frac{\left(1-\mathrm{f}_{\mathrm{c}}\right)}{\left(1-\mathrm{f}_{\mathrm{c}}\right)_{\text {tok }} \mathrm{I}}\left\langle\frac{\overrightarrow{\mathrm{B}} \cdot \nabla \psi \times \nabla \mathrm{B}}{\overrightarrow{\mathrm{B}} \cdot \nabla \mathrm{B}}\right\rangle \simeq \frac{\sqrt{\delta}}{\sqrt{\varepsilon} \mathrm{I}}\left\langle\frac{\overrightarrow{\mathrm{B}} \cdot \nabla \psi \times \nabla \mathrm{B}}{\overrightarrow{\mathrm{B}} \cdot \nabla \mathrm{B}}\right\rangle \sim \frac{1}{\mathrm{I}(\psi)}\left\langle\frac{\overrightarrow{\mathrm{B}} \cdot \nabla \psi \times \nabla \mathrm{B}}{\overrightarrow{\mathrm{B}} \cdot \nabla \mathrm{B}}\right\rangle,
$$

where the last two forms assume a dominant $\mathrm{N}$ and $\mathrm{M}$ of amplitude $\delta \sim \mathrm{r} / \mathrm{R}=\varepsilon$. For a quasipoloidally symmetric or, more generally, a quasi-isodynamic omnigeous, surface $\left\langle B J_{\|}\right\rangle=0$.

The Pfirsch-Schlüter current must be consistent with $\nabla \cdot \overrightarrow{\mathbf{J}}=0$ and

$$
\mathrm{J}_{\|}=\mathrm{J}_{\|}^{\mathrm{PS}}+\mathrm{B}\left\langle\mathrm{B} \mathrm{J}_{\|}\right\rangle /\left\langle\mathrm{B}^{2}\right\rangle,
$$

giving

$$
\mathrm{J}_{\|}^{\mathrm{PS}}=-\frac{\mathrm{c}}{\mathrm{B}}\left(\frac{\partial \mathrm{p}_{\mathrm{i}}}{\partial \psi}+\frac{\partial \mathrm{p}_{\mathrm{e}}}{\partial \psi}\right)\left[\left(1-\frac{\mathrm{B}^{2}}{\left\langle\mathrm{~B}^{2}\right\rangle}\right)\left\langle\frac{\overrightarrow{\mathrm{B}} \cdot \nabla \psi \times \nabla \mathrm{B}}{\overrightarrow{\mathrm{B}} \cdot \nabla \mathrm{B}}\right\rangle+\mathrm{W}\right],
$$

where integrating by parts gives

$$
\langle\mathrm{W}\rangle \propto \Sigma \oint \mathrm{dB} \oint \frac{\mathrm{d} \alpha \mathrm{W}}{\overrightarrow{\mathrm{B}} \cdot \nabla \mathrm{B}} \propto \Sigma \oint \mathrm{dBB} \oint \mathrm{d} \alpha \frac{\partial}{\partial \alpha}\left(\frac{1}{\overrightarrow{\mathrm{b}} \cdot \nabla \mathrm{B} \mid}\right) \int_{\overline{\mathrm{B}}}^{\mathrm{B}} \frac{\mathrm{dB}^{\prime}}{\mathrm{B}^{\prime 3}} \mathrm{~F}_{0}^{\prime},
$$

which indeed vanishes for an omnigenous device as required by Eqs. (7.15), (7.17) and (7.18). In addition, $\mathbf{J}_{\|}^{\mathrm{PS}}$ satisfies

$$
\overrightarrow{\mathrm{B}} \cdot \nabla\left(\frac{\mathrm{J}_{\|}^{\mathrm{PS}}}{\mathrm{B}}\right)=-\mathrm{c}\left(\frac{\partial \mathrm{p}_{\mathrm{i}}}{\partial \psi}+\frac{\partial \mathrm{p}_{\mathrm{e}}}{\partial \psi}\right) \overrightarrow{\mathrm{B}} \cdot \nabla\left(\frac{1}{\mathrm{~B}^{2}}\left\langle\frac{\overrightarrow{\mathrm{B}} \cdot \nabla \psi \times \nabla \mathrm{B}}{\overrightarrow{\mathrm{B}} \cdot \nabla \mathrm{B}}\right\rangle+\frac{W}{\mathrm{~B}^{2}}\right)=-\nabla \cdot\left[\frac{\mathrm{c}}{\mathrm{B}^{2}} \overrightarrow{\mathrm{B}} \times \nabla\left(\mathrm{p}_{\mathrm{i}}+\mathrm{p}_{\mathrm{e}}\right)\right],
$$

since using (4.5) and (4.6) leads to

$$
\overrightarrow{\mathrm{B}} \cdot \nabla\left(\frac{1}{\mathrm{~B}^{2}}\left\langle\frac{\overrightarrow{\mathrm{B}} \cdot \nabla \psi \times \nabla \mathrm{B}}{\overrightarrow{\mathrm{B}} \cdot \nabla \mathrm{B}}\right\rangle+\frac{\mathrm{W}}{\mathrm{B}^{2}}\right)=\overrightarrow{\mathrm{B}} \cdot \nabla\left(\frac{\mathrm{U}}{\mathrm{B}}\right)=\nabla \cdot\left(\frac{1}{\mathrm{~B}^{2}} \overrightarrow{\mathrm{B}} \times \nabla \psi\right) .
$$

So far no collisionality dependence enters the parallel current, unlike the simulations (Beidler et al. 2011; Kernbichler et al.2016) which typically exhibit dependence on $v$ and $\omega$ as $v \rightarrow 0$ for the bootstrap current. The geometric ratio obtained here, using momentum conserving like particle collision operators, is the same as that of the simulations which retain only pitch angle scattering (see Appendix). For more details on differences in coefficients of $\left\langle B J_{\|}\right\rangle$and $\mathrm{V}_{\| \mathrm{i}}$ see the results of Helander et al. (2017) and Landreman et al. (2014). The second reference also shows that at about $\omega \mathrm{R} / \mathrm{v}_{\mathrm{i}} \sim 0.3$ strong electric field effects begin to modify the trapped region of phase space as found by Kagan \& Catto (2010).

The preceding expressions extend the quasisymmetric results of Helander et al. (2017) by having more compact and explicit (though less general) geometric coefficients while retaining tangential drift. They are obtained by a somewhat more general treatment of the $\sqrt{v}$ regime than Helander et al. (2017) which only considers the bootstrap current in the Lorentz collision operator limit. In adddition to the $\sqrt{v}$ regime, the results presented here are valid for superbanana plateau regime transport as only the portion of the perturbed distribution function that is even in $v_{\|}$is altered for it. They reduce to the omnigenous limit (Landreman \& Catto 2012) which is consistent with other earlier results (Shaing et al. 1989; Helander et al. 2011). 
Collisional modifications in the presence of only a tangential $\vec{E} \times \vec{B}$ drift as employed in most simulations are evaluated in the next section. This spurious drive is implied by the right side shown in (6.8). In the next section it will be shown to modify the ion flow and bootstrap current at weak collisionality. At finite $\vec{E} \times \vec{B}$ tangential drift, it offers a possible explanation for some of the discrepancy between simulations and the bootstrap result of (7.15).

\section{A spurious non-omnigenous collisional tangential drift modification}

At small collisionality and finite tangential $\overrightarrow{\mathrm{E}} \times \overrightarrow{\mathrm{B}}$ drift, when the approximate drive term on the right side of (6.8) is retained as in most simulations, a specious ion flow and bootstrap current is generated as will be shown in this section. In this limit an additional contribution to $h_{p}$, defined as $h_{p}^{\omega / v}$, is found by solving the equation suggested by (6.8), namely

$$
\omega \frac{\partial \mathrm{f}_{0}}{\partial \psi}\left\langle\frac{\mathrm{B} \partial \Delta_{\mathrm{p}}}{\mathrm{v}_{\|} \partial \alpha}\right\rangle=\omega \frac{\partial \mathrm{f}_{0}}{\partial \psi}\left(\left\langle\frac{\mathrm{B} \partial}{\mathrm{v}_{\|} \partial \alpha}\left(\frac{\mathrm{v}_{\|}}{\Omega} \frac{\partial \mathrm{F}_{0}}{\partial \alpha}-\frac{\partial \tilde{\mathrm{J}}}{\partial \alpha}\right)\right\rangle=\left\langle\frac{\mathrm{B}}{\mathrm{V}_{\|}} \mathrm{C}\left\{\mathrm{h}_{\mathrm{p}}^{\omega / v}\right\}\right\rangle,\right.
$$

with $\mathrm{h}_{\mathrm{p}}^{\omega / v}=\overline{\mathrm{H}}_{\mathrm{p}}^{\omega / v}$. Inserting $\tilde{\mathbf{J}}$

$$
\frac{2 v \mathrm{QB}_{0} \partial}{\mathrm{v}^{2} \partial \lambda}\left[\lambda\left\langle\mathrm{v}_{\|} \frac{\partial}{\partial \lambda}\left(\mathrm{h}_{\mathrm{p}}^{\omega / v}-\frac{\mathrm{M}_{\mathrm{i}}}{\mathrm{T}} \mathrm{uv}_{\|} \mathrm{f}_{0}\right)\right\rangle\right]=\frac{\omega \mathrm{B}_{0} \partial \mathrm{f}_{0}}{\Omega_{0} \partial \psi}\left\{\left\langle\frac{\partial^{2} \mathrm{~F}_{0}}{\partial \alpha^{2}}\right\rangle-\left\langle\frac{\partial^{2}}{\partial \alpha^{2}}\left[\frac{\mathrm{B}}{\sqrt{1-\lambda \mathrm{B} / \mathrm{B}_{0}}} \int_{\overline{\mathrm{B}}}^{\mathrm{B}} \mathrm{dB} \mathrm{B}^{\prime} \frac{\sqrt{1-\lambda \mathrm{B}^{\prime} / \mathrm{B}_{0}}}{\overrightarrow{\mathrm{b}}^{\prime} \cdot \nabla \mathrm{B}^{\prime}}\right]\right\rangle\right\} .
$$

The right side can be written as a $\lambda$ derivative by using Gradshteyn $\&$ Ryzhik (2007):

$$
\begin{aligned}
& \frac{\sqrt{1-\lambda b^{\prime}}}{\sqrt{1-\lambda b}}=\frac{1-\lambda b^{\prime}}{\sqrt{1-\lambda\left(b+b^{\prime}\right)+\lambda^{2} b b^{\prime}}}= \\
& \frac{\partial}{\partial \lambda}\left\{\left(\frac{b-b^{\prime}}{2 b \sqrt{b b^{\prime}}}\right) \ln \left[2 \sqrt{b^{\prime}(1-\lambda b)\left(1-\lambda b^{\prime}\right)}+2 b b^{\prime} \lambda-\left(b+b^{\prime}\right)\right]-\frac{\sqrt{(1-\lambda b)\left(1-\lambda b^{\prime}\right)}}{b}\right\}
\end{aligned}
$$

where $b=B / B_{0}$ and $b^{\prime}=B^{\prime} / B_{0}$. Integrating once from $\lambda=0$ gives

$$
\begin{aligned}
& \lambda\left(\left\langle\mathrm{v}_{\|}\right\rangle \frac{\partial \mathrm{h}_{\mathrm{p}}^{\omega / v}}{\partial \lambda}+\frac{\mathrm{M}_{\mathrm{i}} \mathrm{v}^{2} \mathrm{f}_{0}}{2 \mathrm{~TB}_{0}}\langle\mathrm{Bu}\rangle\right)=\frac{\omega \mathrm{B}_{0} \mathrm{v}^{2} \partial \mathrm{f}_{0}}{2 \Omega_{0} v \mathrm{Q} \partial \psi}\left\{\frac{\lambda}{\mathrm{B}_{0}}\left\langle\frac{\partial^{2} \mathrm{~F}_{0}}{\partial \alpha^{2}}\right\rangle-\left\langle\frac{\partial^{2}}{\partial \alpha^{2}} \int_{\overline{\mathrm{B}}}^{\mathrm{B}} \frac{\mathrm{dB}^{\prime}}{\overrightarrow{\mathrm{b}}^{\prime} \cdot \nabla \mathrm{B}^{\prime}}\left\{1-\frac{\mathrm{B}^{\prime}-\mathrm{B}^{\prime}}{2 \sqrt{\mathrm{BB}^{\prime}}} \ell \mathrm{n}\left[\frac{2 \sqrt{\mathrm{BB}^{\prime}}-\mathrm{B}^{\prime}-\mathrm{B}^{\prime}}{\mathrm{B}_{0}}\right]\right\}\right\rangle\right. \\
& +\left\langle\frac{\partial^{2}}{\partial \alpha^{2}} \int_{\overline{\mathrm{B}}}^{\mathrm{B}} \frac{\mathrm{dB^{ \prime }}}{\overrightarrow{\mathrm{b}}^{\prime} \cdot \nabla \mathrm{B}^{\prime}}\left\{\sqrt{(1-\lambda \mathrm{b})\left(1-\lambda \mathrm{b}^{\prime}\right)}-\left(\frac{\mathrm{B}-\mathrm{B}^{\prime}}{2 \sqrt{\mathrm{BB}^{\prime}}}\right) \ln \left\{2 \sqrt{\mathrm{bb}^{\prime}(1-\lambda \mathrm{b})\left(1-\lambda \mathrm{b}^{\prime}\right)}+2 b b^{\prime} \lambda-\left(\mathrm{b}+\mathrm{b}^{\prime}\right)\right\}\right\rangle\right\}
\end{aligned}
$$

Neglecting $\mathrm{B}-\mathrm{B}^{\prime}$ terms as small in $\delta$ to simplify the analysis, for the moment, gives

$$
\left.\frac{\left\langle\mathrm{v}_{\|}\right\rangle_{\mathrm{p}} \partial \mathrm{h}_{\mathrm{p}}^{\omega / v}}{\mathrm{f}_{0} \partial \lambda}+\frac{\mathrm{M}_{\mathrm{i}} \mathrm{v}^{2} \mathrm{f}_{0}}{2 \mathrm{~TB} \mathrm{~B}_{0}}\langle\mathrm{Bu}\rangle \simeq \frac{\omega \mathrm{v}^{2} \partial \mathrm{f}_{0}}{2 \Omega_{0} v \mathrm{Q} \partial \psi}\left\langle\frac{\partial^{2}}{\partial \alpha^{2}}\left[\mathrm{~F}_{0}-\frac{\mathrm{B}_{0}}{\lambda} \int_{\overline{\mathrm{B}}}^{\mathrm{B}} \frac{\mathrm{dB}^{\prime}}{\overrightarrow{\mathrm{b}}^{\prime} \cdot \nabla \mathrm{B}^{\prime}}\left(1-\frac{\mathrm{v}_{\|} \mathrm{v}_{\|}^{\prime}}{\mathrm{v}^{2}}\right)\right]\right\rangle\right\}
$$

where now

$$
\mathrm{u}=\left(3 \mathrm{~T} \int \mathrm{d}^{3} \mathrm{vQ} \mathrm{v}_{\|} \mathrm{h}_{\mathrm{p}}^{\omega / v}\right) /\left(\mathrm{M}_{\mathrm{j}} \int \mathrm{d}^{3} \mathrm{vQ} \mathrm{v}^{2} \mathrm{f}_{0}\right)=-\left(3 \mathrm{~T} \int \mathrm{d}^{3} \mathrm{vQ} \mathrm{v}_{\|} \lambda \partial \mathrm{h}_{\mathrm{p}}^{\omega / v} / \partial \lambda\right) /\left(\mathrm{M}_{\mathrm{j}} \int \mathrm{d}^{3} \mathrm{vQ} \mathrm{v}^{2} \mathrm{f}_{0}\right) .
$$

A second integration (making $h_{\mathrm{p}}^{\omega / v}$ vanish at the trapped-passing boundary) is not needed. Notice that $\mathrm{h}_{\mathrm{p}}^{\omega / v} \lesssim \delta_{\text {no }} \omega \rho_{\mathrm{pi}} \mathrm{f}_{0} / v_{\mathrm{i}} \mathrm{a}$ exhibits $\omega / v$ behavior when $\omega>>v_{\mathrm{i}}$, but no $\sqrt{v}$ dependence. For the estimates of this section $\Omega^{-1} \mathrm{~V}_{\|} \partial \mathrm{F}_{0} / \partial \alpha \sim \partial \tilde{\mathrm{J}} / \partial \alpha$ and $\mathrm{F}_{0} \lesssim \mathrm{RB}$ are allowed.

To determine the $h_{p}^{\omega / v}$ modification in the bootstrap current requires evaluating new parallel ion flow velocity contribution $\mathrm{V}_{\mathrm{li}}^{\mathrm{\omega} / \mathrm{v}}$ defined by 


$$
\begin{aligned}
& \mathrm{n}_{\mathrm{i}}\left\langle\mathrm{B} V_{\| \mathrm{li}}^{\omega / v}\right\rangle \equiv\left\langle\mathrm{B} \int \mathrm{d}^{3} \mathrm{vv}_{\|} \mathrm{h}_{\mathrm{p}}^{\omega / v}\right\rangle_{\mathrm{p}}=-\left\langle\frac{\mathrm{B}_{0}^{2}}{\mathrm{~B}} \int \mathrm{d}^{3} \mathrm{vv}_{\|} \lambda \frac{\partial \mathrm{h}_{\mathrm{p}}^{\omega / v}}{\partial \lambda}\right\rangle_{\mathrm{p}}=\frac{\mathrm{M}_{\mathrm{i}}\langle\mathrm{Bu}\rangle}{2 \mathrm{~T}}\left\langle\frac{\mathrm{B}_{0}}{\mathrm{~B}} \int \mathrm{d}^{3} \mathrm{v} \frac{\mathrm{v}_{\|} \lambda \mathrm{v}^{2}}{\left\langle\mathrm{v}_{\|}\right\rangle_{\mathrm{p}}} \mathrm{f}_{0}\right\rangle_{\mathrm{p}} \\
& -\frac{\omega \mathrm{B}_{0}^{2}}{2 v \Omega_{0}}\left\langle\int \mathrm{d}^{3} \mathrm{v} \frac{\mathrm{v}_{\|} \lambda \mathrm{v}^{2} \partial \mathrm{f}_{0}}{\left\langle\mathrm{v}_{\|}\right\rangle_{\mathrm{p}} \mathrm{Q} \partial \psi}\left\{\frac{\partial^{2}}{\partial \alpha^{2}}\left[\mathrm{~F}_{0}-\frac{\mathrm{B}_{0}}{\lambda} \int_{\mathrm{B}}^{\mathrm{B}} \frac{\mathrm{dB}^{\prime}}{\overrightarrow{\mathrm{b}}^{\prime} \cdot \nabla \mathrm{B}^{\prime}}\left(1-\frac{\mathrm{v}_{\|} \mathrm{v}_{\|}^{\prime}}{\mathrm{v}^{2}}\right)\right]\right\}_{\mathrm{p}}\right.
\end{aligned} .
$$

Using $\mathrm{d}^{3} \mathrm{v} \rightarrow 2 \pi\left(\mathrm{Bv}^{3} / \mathrm{B}_{0}\left|\mathrm{v}_{\|}\right|\right) d v d \lambda$, recalling (7.6), and defining the velocity space average

$$
\left\{\mathrm{Q}^{\mathrm{k}}\right\} \equiv\left(\mathrm{M}_{\mathrm{j}} / 3 \mathrm{p}_{\mathrm{j}}\right) \int \mathrm{d}^{3} \mathrm{v} \mathrm{v}^{2} \mathrm{Q}^{\mathrm{k}} \mathrm{f}_{0}=\left(\int_{0}^{\infty} \mathrm{dx} \mathrm{Q}^{\mathrm{k}} \mathrm{x}^{4} \mathrm{e}^{-\mathrm{x}^{2}}\right) /\left(\int_{0}^{\infty} \mathrm{d} \mathrm{xx}^{4} \mathrm{e}^{-\mathrm{x}^{2}}\right)
$$

with $\mathrm{k}=-1,0$, and +1 , gives

$$
\left\langle\frac{B_{0}}{B} \int d^{3} v \frac{v_{\|} \lambda v^{2} f_{0}}{\left\langle v_{\|}\right\rangle_{p}}\right\rangle_{p}=2 \pi \int_{0}^{\infty} d v v^{4} f_{0} \int_{0}^{B_{0} / \vec{B}} \frac{d \lambda \lambda}{\left\langle\sqrt{1-\lambda B / B_{0}}\right\rangle_{p}}=\frac{2}{3} f_{c} \int d^{3} v^{2} f_{0}=\frac{2 f_{c} p_{j}}{M_{j}},
$$

and

$$
\begin{aligned}
& \left\langle\frac{\mathrm{B}_{0}}{\mathrm{~B}} \int \mathrm{d}^{3} \mathrm{v} \frac{\mathrm{v}_{\|} \lambda \mathrm{v}^{2}}{\left\langle\mathrm{v}_{\|}\right\rangle_{\mathrm{p}} \mathrm{Q}} \frac{\partial \mathrm{f}_{0}}{\partial \psi}\right\rangle_{\mathrm{p}}=2 \pi \int_{0}^{\infty} \mathrm{dv} \frac{\mathrm{v}^{4}}{\mathrm{Q}} \frac{\partial \mathrm{f}_{0}}{\partial \psi} \int_{0}^{\mathrm{B}_{0} / \mathrm{B}} \frac{\mathrm{d} \lambda \lambda}{\left\langle\sqrt{1-\lambda \mathrm{B} / \mathrm{B}_{0}}\right\rangle_{\mathrm{p}}}=\frac{2}{3} \mathrm{f}_{\mathrm{c}} \int \mathrm{d}^{3} \mathrm{v} \frac{\mathrm{v}^{2} \partial \mathrm{f}_{0}}{\mathrm{Q} \partial \psi} . \\
& =\frac{2 \mathrm{f}_{\mathrm{c}}}{\mathrm{M}_{\mathrm{j}}}\left[\left\{\frac{1}{\mathrm{Q}}\right\}\left(\frac{\partial \mathrm{p}_{\mathrm{j}}}{\partial \psi}+\operatorname{Zen} \frac{\partial \Phi}{\partial \psi}\right)+\left\{\frac{\mathrm{M}_{\mathrm{j}} \mathrm{v}^{2}-5 \mathrm{~T}}{2 \mathrm{TQ}}\right\} \mathrm{n} \frac{\partial \mathrm{T}}{\partial \psi}\right]
\end{aligned}
$$

To obtain an analytically tractable estimate the last term in (8.7) is simplified further by using the freely passing expansion $\lambda^{-1} B_{0}\left(1-\mathrm{v}^{-2} \mathrm{v}_{\|} \mathrm{v}_{\|}\right) \simeq\left(\mathrm{B}+\mathrm{B}^{\prime}\right) / 2$ for now. Then the resulting approximation can be added to $\mathrm{F}_{0}$ and treated in the same way by using

$$
\mathrm{G}_{0} \equiv \mathrm{F}_{0}-\lambda^{-1} \mathrm{~B}_{0} \int_{\overline{\mathrm{B}}}^{\mathrm{B}} \frac{\mathrm{dB}^{\prime}}{\overrightarrow{\mathrm{b}}^{\prime} \cdot \nabla \mathrm{B}^{\prime}}\left(1-\frac{\mathrm{v}_{\|} \mathrm{v}_{\|}^{\prime}}{\mathrm{v}^{2}}\right) \simeq \mathrm{F}_{0}-\frac{1}{2} \int_{\overline{\mathrm{B}}}^{\mathrm{B}} \frac{\mathrm{dB}^{\prime}\left(\mathrm{B}^{\prime}+\mathrm{B}\right)}{\overrightarrow{\mathrm{b}}^{\prime} \cdot \nabla \mathrm{B}^{\prime}},
$$

where the fact that lower limit of the $\mathrm{F}_{0}$ integral is $\mathrm{B}_{0}$, and not $\overline{\mathrm{B}}=\widehat{\mathrm{B}}$, is unimportant since only $\alpha$ derivatives of $\mathrm{G}_{0}$ enter.

Also needed is (8.6) which gives

$$
\langle\mathrm{Bu}\rangle\left[\int \mathrm{d}^{3} \mathrm{vQv} \mathrm{v}^{2} \mathrm{f}_{0}-\frac{3}{2}\left\langle\frac{\mathrm{B}_{0}}{\mathrm{~B}} \int \mathrm{d}^{3} \mathrm{v} \frac{\mathrm{v}_{\|} \lambda \mathrm{v}^{2}}{\left\langle\mathrm{v}_{\|}\right\rangle_{\mathrm{p}}} \mathrm{Qf}_{0}\right\rangle_{\mathrm{p}}\right]=-\frac{3 \mathrm{~B}_{0} \omega \mathrm{T}}{2 \mathrm{M}_{\mathrm{i}} v}\left\langle\int \mathrm{d}^{3} \mathrm{v} \frac{\lambda \mathrm{v}^{2} \partial \mathrm{f}_{0} \partial \Delta_{\mathrm{p}}}{\left\langle\mathrm{v}_{\|}\right\rangle_{\mathrm{p}} \partial \psi \partial \alpha}\right\rangle_{\mathrm{p}},
$$

where

$$
\left\langle\frac{\mathrm{B}_{0}}{\mathrm{~B}} \int \mathrm{d}^{3} \mathrm{v} \frac{\mathrm{v}_{\|} \lambda \mathrm{v}^{2} \mathrm{Qf}_{0}}{\left\langle\mathrm{v}_{\|}\right\rangle_{\mathrm{p}}}\right\rangle_{\mathrm{p}}=2 \pi \int_{0}^{\infty} \mathrm{dvv}^{4} \mathrm{Qf}_{0} \int_{0}^{\mathrm{B}_{0} / \hat{\mathrm{B}}} \frac{\mathrm{d} \lambda \lambda}{\left\langle\sqrt{1-\lambda \mathrm{B} / \mathrm{B}_{0}}\right\rangle_{\mathrm{p}}}=\frac{2}{3} \mathrm{f}_{\mathrm{c}} \int \mathrm{d}^{3} \mathrm{vv}^{2} \mathrm{Qf}_{0} \equiv \frac{2 \mathrm{f}_{\mathrm{c}} \mathrm{p}_{\mathrm{j}}}{\mathrm{M}_{\mathrm{j}}}\{\mathrm{Q}\} .
$$

Also, noticing that

$$
\left\langle\frac{\mathrm{B}_{0}}{\mathrm{~B}} \int \mathrm{d}^{3} \mathrm{v} \frac{\mathrm{v}_{\|} \lambda \mathrm{v}^{2}}{\left\langle\mathrm{v}_{\|}\right\rangle_{\mathrm{p}}} \frac{\partial \mathrm{f}_{0}}{\partial \psi}\right\rangle_{\mathrm{p}}=2 \pi \int_{0}^{\infty} \mathrm{dvv}^{4} \frac{\partial \mathrm{f}_{0}}{\partial \psi} \int_{0}^{\mathrm{B}_{0} \hat{\mathrm{B}}} \frac{\mathrm{d} \lambda \lambda}{\left\langle\sqrt{1-\lambda \mathrm{B} / \mathrm{B}_{0}}\right\rangle_{\mathrm{p}}}=\frac{2}{3} \mathrm{f}_{\mathrm{c}} \int \mathrm{d}^{3} \mathrm{vv}^{2} \frac{\partial \mathrm{f}_{0}}{\partial \psi}=\frac{2 \mathrm{f}_{\mathrm{c}}}{\mathrm{M}_{\mathrm{i}}}\left(\frac{\partial \mathrm{p}}{\partial \psi}+\operatorname{Zen} \frac{\partial \Phi}{\partial \psi}\right) .
$$

gives upon using (8.12)

$$
\left\langle\frac{\mathrm{B}_{0}}{\mathrm{~B}} \int \mathrm{d}^{3} \mathrm{v} \frac{\mathrm{v}_{\|} \lambda \mathrm{v}^{2} \partial \mathrm{f}_{0} \partial^{2} \mathrm{G}_{0}}{\left\langle\mathrm{v}_{\|}\right\rangle_{\mathrm{p}} \partial \psi \partial \alpha^{2}}\right\rangle_{\mathrm{p}} \simeq \frac{2 \mathrm{f}_{\mathrm{c}}}{\mathrm{M}_{\mathrm{i}}}\left\langle\frac{\partial^{2} \mathrm{G}_{0}}{\partial \alpha^{2}}\right\rangle\left(\frac{\partial \mathrm{p}}{\partial \psi}+\operatorname{Zen} \frac{\partial \Phi}{\partial \psi}\right),
$$

Substituting in yields 


$$
\langle\mathrm{Bu}\rangle=\frac{-\mathrm{f}_{\mathrm{c}} \mathrm{c} \omega}{\operatorname{Ze}\left(1-\mathrm{f}_{\mathrm{c}}\right) v \mathrm{n}\{\mathrm{Q}\}}\left\langle\frac{\partial^{2} \mathrm{~F}_{0}}{\partial \alpha^{2}}\right\rangle\left(\frac{\partial \mathrm{p}}{\partial \psi}+\operatorname{Zen} \frac{\partial \Phi}{\partial \psi}\right) \lesssim \mathrm{B} \frac{\delta_{\mathrm{no}} \omega \rho_{\mathrm{pi}} \mathrm{v}}{v_{\mathrm{i}} \mathrm{a}} .
$$

The collisional bootstrap modification $\left\langle\mathrm{BJ}_{\|}^{\omega / v}\right\rangle$ to be added to (7.15) is then

$$
\left\langle\mathrm{BJ}_{\|}^{\omega / v}\right\rangle \equiv \operatorname{Zen}_{\mathrm{i}}\left\langle\mathrm{BV}_{\| \mathrm{i}}^{\omega / v}\right\rangle=\frac{\mathrm{cf}_{\mathrm{c}} \omega}{\left(1-\mathrm{f}_{\mathrm{c}}\right) v_{\mathrm{i}}}\left\langle\frac{\partial^{2} \mathrm{G}_{0}}{\partial \alpha^{2}}\right\rangle\left[\left(\left\{\frac{1-\mathrm{f}_{\mathrm{c}}}{\mathrm{Q}}\right\}-\frac{\mathrm{f}_{\mathrm{c}}}{\{\mathrm{Q}\}}\right)\left(\frac{\partial \mathrm{p}_{\mathrm{i}}}{\partial \psi}+\operatorname{Zen}_{\mathrm{i}} \frac{\partial \Phi}{\partial \psi}\right)+\left(1-\mathrm{f}_{\mathrm{c}}\right)\left\{\frac{\mathrm{M}_{\mathrm{i}} \mathrm{v}^{2}-5 \mathrm{~T}_{\mathrm{i}}}{2 \mathrm{~T}_{\mathrm{i}} \mathrm{Q}}\right\} \mathrm{n}_{\mathrm{i}} \frac{\partial \mathrm{T}_{\mathrm{i}}}{\partial \psi}\right],
$$

where $\left\langle\partial^{2} \mathrm{G}_{0} / \partial \alpha^{2}\right\rangle$ as given by (8.12) only vanishes for an omnigenous flux surface, and $\{\mathrm{Q}\}=0.4$ and $\left\{\mathrm{Q}^{-1}\right\}=5.4$ (Catto et al. 2001). Upon dividing by $\mathrm{Ze}, \mathrm{n}_{\mathrm{i}}\left\langle\mathrm{BV}_{\mathrm{li}}^{\omega}\right\rangle$ is to be added to the parallel ion velocity expression (7.13). Forming the ratio of (8.17) divided by (7.15) gives

$$
\frac{\left\langle\mathrm{BJ}_{\|}^{\omega / v}\right\rangle}{\left\langle\mathrm{BJ}_{\|}\right\rangle} \sim \frac{\delta_{\mathrm{no}} \omega}{\delta v_{\mathrm{i}}},
$$

and therefore a specious modification of the bootstrap current. This modification should be present in simulations that use the radially local form of the drift kinetic equation and does not, in general, vanish on imperfectly quasi-poloidally symmetric or quasi-isodynamic surfaces.

To remove the approximation (8.11)-(8.12) in (8.17) the substitution

$$
\mathrm{G}_{0} \Rightarrow \mathrm{F}_{0}-\left\langle\int \mathrm{d}^{3} \mathrm{v} \frac{\mathrm{v}_{\|} \mathrm{v}^{2} \partial \mathrm{f}_{0}}{\left\langle\mathrm{v}_{\|}\right\rangle_{\mathrm{p}} \partial \psi_{\overline{\mathrm{B}}}^{\mathrm{B}}} \int_{\mathrm{b}^{\prime} \cdot \nabla \mathrm{B}^{\prime}}\left(1-\frac{\mathrm{v}_{\|} \mathrm{v}_{\|}^{\prime}}{\mathrm{v}^{2}}\right)\right\rangle_{\mathrm{p}} /\left\langle\int \mathrm{d}^{3} \mathrm{v} \frac{\mathrm{v}_{\|} \lambda \mathrm{v}^{2} \partial \mathrm{f}_{0}}{\mathrm{~B}\left\langle\mathrm{v}_{\|}\right\rangle_{\mathrm{p}} \partial \psi}\right\rangle_{\mathrm{p}},
$$

is required. A full generalization can be made similarly by retaining the $\mathrm{B}-\mathrm{B}^{\prime}$ terms of (8.4).

The spurious collisional, non-omnigenous, tangential $\overrightarrow{\mathrm{E}} \times \overrightarrow{\mathrm{B}}$ drift modification is more important for the ions than the electrons, and dominates for $\omega \delta_{n o} / v_{i}>>$. It introduces unphysical radial electric field dependence through both the tangential drift frequency $\omega$ and a new $\partial \Phi / \partial \psi$ force term in (8.17) so that both $\omega$ and $\omega^{2}$ terms enter. It is perhaps responsible for some of the $\partial \Phi / \partial \psi$ dependence observed in simulations at small collisionalities (Beidler et al. 2011). However, it does not explain the descrepancy between analytical and numerical results as $\omega=\mathrm{c} \partial \Phi / \partial \psi \rightarrow 0$. This behavior must be due to the transitional particles that spend time in multiple wells and are present, at least to some degree, in all the configurations considered in Beidler et al. (2011). The evaluation of the transport and parallel flow associated with these transitional particles are beyond the scope of the treatment presented here.

The new term considered in this section results in no fluxes from $\left\langle\int \mathrm{d}^{3} \mathrm{vh}_{\mathrm{p}}^{\omega / v} \overrightarrow{\mathrm{v}}_{\mathrm{d}} \cdot \nabla \psi\right\rangle$ and $\left\langle\int \mathrm{d}^{3} \mathrm{vh}_{\mathrm{p}}^{\omega / v}\left(\mathrm{M}_{\mathrm{i}} \mathrm{v}^{2} / 2\right) \overrightarrow{\mathrm{v}}_{\mathrm{d}} \cdot \nabla \psi\right\rangle$, and no frictional particle flux as $\int \mathrm{d}^{3} \mathrm{vv}_{\|} \mathrm{C}\left\{\mathrm{h}_{\mathrm{p}}^{\omega / v}\right\}=0$ for ion-ion collisions; however, it will give rise to a tangential $\overrightarrow{\mathrm{E}} \times \overrightarrow{\mathrm{B}}$ drift dependent, but collision frequency independent heat flux, $\left\langle\int \mathrm{d}^{3} \mathrm{vh}_{\mathrm{p}}^{\omega / v}\left(\mathrm{Mv}^{2} / 2\right) \overrightarrow{\mathrm{v}} \cdot \nabla \psi\right\rangle=(\mathrm{Mc} / \mathrm{Ze})\left\langle\mathrm{U} \int \mathrm{d}^{3} \mathrm{v}\left(\mathrm{Mv}^{2} / 2\right) \mathrm{v}_{\|} \mathrm{C}\left\{\mathrm{h}_{\mathrm{p}}^{\omega / v}\right\}\right\rangle$. To see there is such a flux, the solenoidal vector $\overrightarrow{\mathrm{h}}=\mathrm{B}^{-2} \overrightarrow{\mathrm{B}} \times \nabla \psi+\mathrm{U} \overrightarrow{\mathrm{b}}$ is introduced (Simakov \& Helander 2009) and the $\overrightarrow{\mathrm{h}} \cdot \overrightarrow{\mathrm{v}} \mathrm{v}^{2} / 2$ moment of the Fokker-Planck equation formed. The absence of an impact on the radial particle flux is consistent with the Beidler et al. (2011) simulations. 


\section{Discussion}

The streamlined derivation of the bootstrap current and the parallel ion velocity presented here is possible because the problem is formulated in a way that allows all of the odd terms in $\mathrm{v}_{\|}$ to be obtained by evaluating them as part of the leading order corrections to the Maxwellian. As a result, the only other terms that enter in lowest order are trapped terms and they are all even in $\mathrm{V}_{\|}$. The procedure results in a parallel ion flow velocity (7.9) consistent with force balance and continuity, and leads to convenient expressions for the Pfirsch-Schlüter parallel ion velocity (7.14) and parallel current density (7.18). The bootstrap current (7.15) and bootstrap contribution to the parallel ion velocity (7.13) have compact and explicit geometric coefficients that agree with the previous collisionless forms of Helander et al. (2017 \& 2011) and Landreman \& Catto (2012), which are both consistent with Shaing et al. (1989). As the Helander et al. (2017) bootstrap current expression is derived in the Lorentz collision operator limit for the $\sqrt{v}$ regime, the result presented here, (7.15), is the most general expression derived to date as it is valid in the $1 / v, \sqrt{v}$, and superbanana plateau regimes.

Perhaps more importantly, a new, but spurious, collisional modification to the ion parallel flow velocity and bootstrap current given by (8.17) and (8.19) (or its further generalization) is found that can become substantial at low collisionalities whenever there is a tangential $\vec{E} \times \overrightarrow{\mathrm{B}}$ drift and the magnetic field is not perfectly omnigenous. In addition to the dependence of (8.17) on the tangential drift frequency, $\omega=\mathrm{c} \partial \Phi / \partial \psi$, there is a new force term depending on $\partial \Phi / \partial \psi$, so terms both linear and quadratic in $\omega$ occur so exhibits a sensitivity to $\partial \Phi / \partial \psi$ as observed in the simulations. This new $\omega / v_{i}$ contribution should be present in numerical solutions of the drift kinetic equation and may be a part of the reason these sometimes do not agree with previous analytical expressions. Unfortunately, it does not explain the descrepancy between analytical and numerical results as $\omega=\mathrm{c} \partial \Phi / \partial \psi \rightarrow 0$. The disagreement in this limit is presumably due to transitional particles that de-trap and re-trap in multiple wells and act to widen the boundary layer width between at the trapped-passing boundary. These transitional particles are expected to arise when the departure from omnigeneity becomes significant. However, the spurious $\omega / v_{i}$ contribution is an artifact of the radially local approximation of the drift kinetic equation that is usually employed in analytical theory as well as in neoclassical codes. It disappears when the effect of the full drift velocity on the perturbed distribution function is retained in the kinetic equation. This result is yet another indication that it is not always logically consistent to treat neoclassical transport in stellarators as a radially local process.

At low collisionalities, estimates indicate that the transitional ions widen the trappedpassing boundary layer to introduce a regime linear in $v_{*}$ (Beidler et al. 2011; Catto 2019) that cannot be treated by the procedures herein.

\section{Acknowledgments}

The work was supported the US Department of Energy grant DE-FG02-91ER-54109.

\section{Appendix: Lorentz forms}


The Lorentz operator form of the results for (8.17) and (7.15) are

and

$$
\left\langle\mathrm{BJ}_{\|}^{\omega / v}\right\rangle_{\mathrm{L}} \equiv \operatorname{Zen}_{\mathrm{i}}\left\langle\mathrm{BV}_{\| \mathrm{i}}^{\omega / v}\right\rangle_{\mathrm{L}}=\frac{\mathrm{cf}_{\mathrm{c}} \omega}{v_{\mathrm{i}}}\left\langle\frac{\partial^{2} \mathrm{G}_{0}}{\partial \alpha^{2}}\right\rangle\left[\left\{\frac{1}{\mathrm{Q}}\right\}\left(\frac{\partial \mathrm{p}_{\mathrm{i}}}{\partial \psi}+\operatorname{Zen}_{\mathrm{i}} \frac{\partial \Phi}{\partial \psi}\right)+\left\{\frac{\mathrm{M}_{\mathrm{i}} \mathrm{v}^{2}-5 \mathrm{~T}_{\mathrm{i}}}{2 \mathrm{~T}_{\mathrm{i}} \mathrm{Q}}\right\} \mathrm{n}_{\mathrm{i}} \frac{\partial \mathrm{T}_{\mathrm{i}}}{\partial \psi}\right],
$$

$$
\left\langle\mathrm{BJ}_{\|}\right\rangle_{\mathrm{L}}=\mathrm{c}\left(1-\mathrm{f}_{\mathrm{c}}\right)\left(\frac{\partial \mathrm{p}_{\mathrm{i}}}{\partial \psi}+\operatorname{Zen}_{\mathrm{i}} \frac{\partial \Phi}{\partial \psi}\right)\left\langle\frac{\overrightarrow{\mathrm{B}} \cdot \nabla \psi \times \nabla \mathrm{B}}{\overrightarrow{\mathrm{B}} \cdot \nabla \mathrm{B}}\right\rangle+\left\langle\mathrm{BJ}_{\|}^{\omega / v}\right\rangle_{\mathrm{L}}
$$

\section{References}

Anderson, F., Almagri, A., Anderson, D., Matthews, P., Talmadge, J. \& Shohet, J. 1995 The Helically Symmetric Experiment, (HSX) goals, design and status. Fusion Technol. 27, 273-277.

Antonsen, T. M. \& CHU, M. S. 1982 Radio frequency current generation by waves in toroidal geometry. Phys. Fluids 25, 1295-1296.

Beidler, C. D., Allmaier, K., Isaev, M. Y., Kasilov, S. V., Kernbichler, W., Leitold, G. O., MAaßBerg, H., MikKelsen, D. R., Murakami, S., Schmidt, M., SPOnG, D. A., TRIBALDOS, V. \& WAKASA, A. 2011 Benchmarking of the mono-energetic transport coefficients - results from the International Collaboration on Neoclassical Transport in Stellarators (ICNTS). Nucl. Fusion 51 (7), 076001 (28pp).

BEIDLER, C. D. \& D'HAESELEER, W. D. 1995 A general solution of the ripple-averaged kinetic equation (GSRAKE). Plasma Phys. Control. Fusion 37, 463-490.

Beidler, C. D., Grieger, G., Hermegger, F., Hameyer, E., Kisslinger, J., Lotz, W., MaAssberg, H., Merkel, P., Nührenberg, J., Rau, F., SapPer, J., SArdei, F., ScARdovelli, R., Schülter, A., \& Wobig, H. 1990 Physics and engineering design for Wendelstein VII-X. Fusion Technol. 17, 148-168.

BoOzER, A. H. 1981 Plasma equilibrium with rational magnetic surfaces. Phys. Fluids 24, 19992003.

Boozer, A. H. 1995 Quasi-helical symmetry in stellarators. Plasma Phys. Control. Fusion 37, A103-117.

Calvo, I., PARra, F. I., Velasco, J. L. \& Alonso, J. A. 2017 The effect of tangential drifts on neoclassical transport in stellarators close to omnigeneity. Plasma Phys. Control. Fusion 59, 055014 (19pp).

CARY, J. R. \& SHASHARINA, S. G. 1997a Omnigenity and quasihelicity in helical plasma confinement systems. Phys. Plasmas 4, 3323-3333.

CARY, J. R. \& SHASHARINA, S. G. 1997b Helical plasma confinement devices with good confinement properties. Phys. Rev. Lett. 78, 674-677.

CATTO, P. J. 2019 Collisional alpha transport in a weakly non-quasisymmetric stellarator magnetic field. J. Plasmas Physics 85, ???.

Catto, P. J., Hastie, R. J., Hutchinson, I. H. \& Helander, P. 2001 Effect of the inductive electric field on ion flow in tokamaks. Phys. Plasmas 8, 3334-3341. 
Connor, J. W., Grimm, R. C., Hastie, R. J. \& KeePing, P. M. 1973 The conductivity of a toroidal plasma. Nuclear Fusion 13, 211-214.

Galeev, A. A., Sagdeev, R. Z., Furth, H. P. \& Rosenbluth, M. N. 1969 Plasma diffusion in a toroidal stellarator. Phys. Rev. Lett. 22, 511-514.

GArren, D. A. \& BoOzer, A. H. 1991 Existence of quasihelically symmetric stellarators. Phys. Fluids B 3, 2822-2834.

Gradshteyn, I. S. \& RYZHIK, I. M. 2007 Table of Integrals, Series, and Products, 7th edn. pp. 93-94. Elsevier/Academic.

Grieger, G., Lotz, W., Merkel, P., Nührenberg, J., Sapper, J., Strumberger, E., Wobig, H., the W7-X Team, Burhenn, V., ErcKmannn, V., Gasparino, U., GiannONe, L., HARTFuss, H. J., JAEniCKe, R., KÜHner, G., Ringler, H., Weller, A., Wagner, K. \& the W7-AS TEAM. 1992 Physics optimization of stellarators. Phys. Fluids B 4, 2081-2091.

HaZEltine, R. D. 1973 Recursive derivation of the drift-kinetic equation. Plasma Physics 15, 77-80.

HELANDER, P. 2014 The theory of plasma confinement in non-axisymmetric magnetic fields. Rep. Progress Phys. 77, 087001 (35 pp).

Helander, P., Geiger, J. \& MAAßBERG, H. 2011 On the bootstrap current in stellarators and tokamaks. Phys. Plasmas 18, 092505 (8pp).

Helander, P. \& NÜHRENBERG, J. 2009 Bootstrap current and neoclassical transport in quasiisodynamic stellarators. Plasma Phys. Control. Fusion 51, 055004 (9pp).

Helander, P., PARra, F. I. \& NeWton, S. L. 2017 Stellarator bootstrap current and plasma flow velocity at low collisionality. J. Plasma Phys. 83, 905830206 (25pp).

Helander, P. \& SigmaR, D. J. Collisional transport in magnetized plasmas (Cambridge University Press, Cambridge, United Kingdom 2002) pp191-195 \& 206-207.

Henneberg, S. A., Drevlak, M, Nührenberg, C., Beidler, C. D., Turkin, Y., Loizu, J. \& HELANDER, P. 2019 Properties of a new quasi-axisymmetric configuration, Nucl. Fusion 59, 026014 (11pp).

Ho, D. D-M. \& KulSRUd, R. M. , 1987 Neoclassical transport in stellarators, Phys. Fluids 30, 442-461.

KAgAn, G. \& CATto, P. J. 2010 Neoclassical ion heat flux and poloidal flow in a tokamak pedestal. Plasma Phys. Control. Fusion 52, 055004 (19pp).

Kernbichler, W., Kasilov, S. V., Kapper, G., Martitsch, A. F., Nemov, V. V., Albert, C. \& HEYN, M. F. 2016 Solution of drift kinetic equation in stellarators and tokamaks with broken symmetry using the code NEO-2. Plasma Phys. Control. Fusion 58, 104001 (15pp).

LAndreman, M. \& CATto, P. J. 2012 Omnigenity as generalized quasisymmetry. Phys. Plasmas 19, 056103 (16pp).

LANDREMAN, M. \& SENGUPTA, W. 2018 Direct construction of optimized stellarator shapes. Part 1. Theory in cylindrical coordinates. J. Plasma Physics 84, 905840616 (22pp). 
Landreman, M., Sengupta, W. \& Plunk, G. G. 2019 Direct construction of optimized stellarator shapes. Part 2. Numerical quasisymmetric solutions. J. Plasma Physics 85, submitted (22pp).

Landreman, M., Smith, H. M., Mollen, A. \& Helander, P. 2014 Comparison of particle trajectories and collision operators for collisional transport in nonaxisymmetric plasmas. Physics Plasmas 21, 042503-16.

MAtSUOKA, SATAKE, KANNO \& SUGAMA, 2015 Effects of magnetic drift tangential to magnetic surfaces on neoclassical transport in non-axisymmetric plasmas. Physics Plasmas 22, 072511-13.

NüHrenberg, J. \& ZILle, R. 1988 Quasi-helically symmetric toroidal stellarators. Phys. Lett. A 129, $113-117$.

NüHrenberg, J. 2010 Development of quasi-isodynamic stellarators. Plasma Phys. Control. Fusion 52, 124003 (7pp).

Plunk, G. G. \& Helander, P. 2018 Quasi-axisymmetric equilibria: weakly non-axisymmetric case in a vacuum. J. Plasma Phys. 84, 905840205 (18pp).

Rosenbluth, M. N., Hazeltine, R. D. \& Hinton, F. L. 1972 Plasma transport in toroidal confinement systems. Physics Fluids 15, 116-140 (see Appendix A).

SHAING, K. C., 2015 Superbanana and superbanana plateau transport in finite aspect ratio tokamaks with broken symmetry. J. Plasma Phys. 81, 905810203; 12pp.

Shaing, K. C., Carreras, B. A., Dominguez, N., Lynch, V. E. \& Tolliver, J. S. 1989 Bootstrap current control in stellarators. Phys. Fluids B 1, 1663-1670.

Simakov, A. N. \& CATTO, P. J. 2005 Drift kinetic equation exact through second order in gyroradius expansion. Phys. Plasmas 12,012105-9.

Simakov, A. N. \& HelAnder, P. 2009 Neoclassical momentum transport in a collisional stellarator and a rippled tokamak. Phys. Plasmas 16, 042503-8. 